\title{
HIGH-RESOLUTION 3D IMAGING OF OSTEOCYTES AND COMPUTATIONAL MODELLING IN MECHANOBIOLOGY: INSIGHTS ON BONE DEVELOPMENT, AGEING, HEALTH AND DISEASE
}

\author{
P.M. Goggin ${ }^{1}$, K.C. Zygalakis ${ }^{2}$, R.O.C. Oreffo ${ }^{3}$ and P. Schneider ${ }^{1 *}$ \\ ${ }^{1}$ Bioengineering Science Research Group, Faculty of Engineering and the Environment, University of Southampton, \\ Southampton, UK \\ ${ }^{2}$ Mathematical Sciences, Faculty of Social, Human and Mathematical Sciences, University of Southampton, \\ Southampton, UK \\ ${ }^{3}$ Bone and Joint Research Group, Centre for Human Development, Stem Cells and Regeneration, Faculty of \\ Medicine, University of Southampton, Southampton, UK
}

\begin{abstract}
Osteocytes are involved in mechanosensation and mechanotransduction in bone and hence, are key to bone adaptation in response to development, ageing and disease. Thus, detailed knowledge of the three-dimensional (3D) structure of the osteocyte network (ON) and the surrounding lacuno-canalicular network (LCN) is essential. Enhanced understanding of the ON\&LCN will contribute to a better understanding of bone mechanics on cellular and sub-cellular scales, for instance through improved computational models of bone mechanotransduction. Until now, the location of the ON within the hard bone matrix and the sub- $\mu \mathrm{m}$ dimensions of the ON\&LCN have posed significant challenges for 3D imaging. This review identifies relevant microstructural phenotypes of the ON\&LCN in health and disease and summarises how light microscopy, electron microscopy and X-ray imaging techniques have been used in studies of osteocyte anatomy, pathology and mechanobiology to date. In this review, we assess the requirements for ON\&LCN imaging and examine the state of the art in the fields of imaging and computational modelling as well as recent advances in high-resolution 3D imaging. Suggestions for future investigations using volume electron microscopy are indicated and we present new data on the ON\&LCN using serial block-face scanning electron microscopy. A correlative approach using these high-resolution 3D imaging techniques in conjunction with in silico modelling in bone mechanobiology will increase understanding of osteocyte function and, ultimately, lead to improved pathways for diagnosis and treatment of bone diseases such as osteoporosis.
\end{abstract}

Keywords: Osteocyte, 3D imaging, microscopy, biomechanics, lacuno-canalicular network, mechanobiology, mechanosensation, mechanotransduction, osteoporosis.

*Address for correspondence:

Dr. Philipp Schneider

Faculty of Engineering and the Environment,

University of Southampton,

Southampton, SO17 1BJ, UK

Telephone number: +44 (0) 2380594640

Email: p.schneider@soton.ac.uk
Table of abbreviations

$\begin{array}{ll}\text { 2D } & \text { 2-dimensional } \\ \text { 3D } & \text { 3-dimensional } \\ \text { AFM } & \text { Atomic force microscopy } \\ \text { ATUM } & \text { Automatic tape-collecting } \\ & \text { ultramicrotome } \\ \text { CLSM } & \text { Confocal laser scanning microscopy } \\ \text { CT } & \text { Computed tomography } \\ \text { ECM } & \text { Extracellular matrix } \\ \text { EM } & \text { Electron microscopy } \\ \text { FE } & \text { Finite element } \\ \text { FSI } & \text { Fluid-structure interaction } \\ \text { LCN } & \text { Lacuno-canalicular network } \\ \text { LM } & \text { Light microscopy } \\ \text { LSM } & \text { Light sheet microscopy } \\ \mu \text { CT } & \text { Micro-computed tomography } \\ \text { ON } & \text { Osteocyte network } \\ \text { PCM } & \text { Pericellular matrix } \\ \text { PCS } & \text { Pericellular space } \\ \text { SBF SEM } & \text { Serial block-face scanning electron } \\ & \text { microscopy } \\ \text { SEM } & \text { Scanning electron microscopy } \\ \text { Serial FIB SEM } & \text { Serial focused ion beam scanning } \\ & \text { electron microscopy } \\ \text { SR CT } & \text { Synchrotron radiation-based computed } \\ \text { TEM } & \text { tomography } \\ \text { TXM } & \text { Transmission electron microscopy } \\ \text { UHVEM } & \text { Transmission X-ray microscopy } \\ & \text { Ultra-high voltage electron microscopy } \\ & \\ & \end{array}$

\section{Introduction}

Through bone adaptation, the skeleton adapts continuously to changed mechanical loading patterns due to development, growth, ageing, disease, disuse or exercise, by removing existing and adding new bone tissue. It has been recognised that osteocytes are the key cells which orchestrate bone adaptation (Tatsumi et al., 2007). Osteocytes are ovoid cells approximately $10 \mu \mathrm{m}$ long, surrounded by a pericellular matrix (PCM). The osteocytes and their processes form the osteocyte network $(\mathrm{ON})$, which is housed within the lacuno-canalicular network ( $\mathrm{LCN})$, a system of voids and channels in the calcified bone matrix (Fig. 1). The presence of the osteocytes throughout the bone tissue and their interconnectedness makes osteocytes ideally placed to 
Fig. 1. Schematic view of the ON\&LCN. The LCN is formed by osteocyte lacunae and interconnecting canaliculi. Image adapted from Schneider et al. (2010) with kind permission of Elsevier.

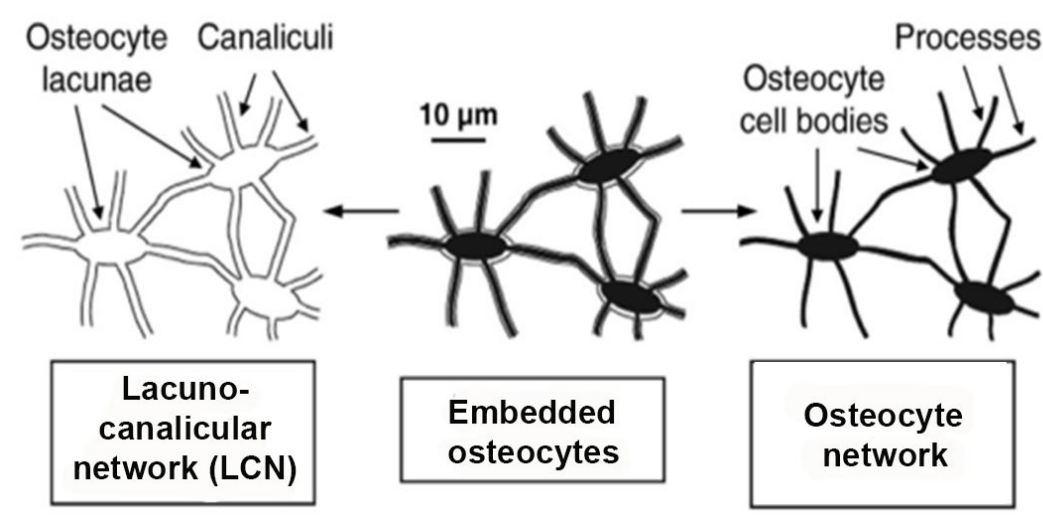

sense changes in mechanical stimuli and to communicate this information through the ON. The osteocyte and the lacuno-canalicular networks (ON\&LCN) are thought to be a mechanosensitive organ for external mechanical stimuli, which are translated into biochemical signals that lead to bone resorption and formation by osteoclasts and osteoblasts, respectively. As such, the ON\&LCN are central in the bone remodelling process. Thus, changes in the local bone microstructure, including the ON\&LCN, can alter and disrupt mechanotransduction and mechanosensation mechanisms, which are implicated in bone diseases such as osteoporosis, osteomalacia/rickets and osteopetrosis (McCreadie et al., 2004; Neve et al., 2012).

Knowledge of the three-dimensional (3D) structure of the ON\&LCN would inform conclusions about the function and malfunction of osteocytes for different bone states in development, ageing, disease, disuse or exercise. More specifically, enhanced knowledge would improve the predictive power and accuracy of computational models, which attempt to elucidate the mechanisms of mechanotransduction using 3D geometries for the ON\&LCN. Recent finite element and fluid-structure interaction models have used relatively low resolution image data and made a priori assumptions about the actual dimensions of the ON\&LCN, such as the pericellular space (PCS) between the ON\&LCN (Verbruggen et al., 2012; Vaughan et al., 2014; Verbruggen et al., 2014). One perspective on osteoporosis is that it is a failure of bone's adaptation to functional loading, that is, a failure of its mechanotransduction and/or mechanosensation capacity (Sharma et al., 2012; Ciani et al., 2014). Two mechanisms, direct response to matrix deformation and indirect response to fluid flow alteration have been suggested as possible pathways for mechanotransduction. To determine in detail how these mechanisms enable load transfer and signal transmission, detailed 3D mapping of the ON\&LCN is needed. The fine structural details of osteocytes and their processes, the PCS and the connections between the cell and the surrounding bone matrix need to be resolved and quantified. However, structural data of the ON\&LCN at cellular and sub-cellular scales of adequate quality for realistic computational modelling approaches for bone mechanotransduction, which is based on experimental data, have not been provided up to now.
This is due to both the inaccessible location of the osteocytes and their processes and to the sub- $\mu \mathrm{m}$ dimensions. Our review will:

1. Identify microstructural phenotypes of the ON\&LCN, relevant for bone development, ageing, health and disease.

2. Attempt to define the ideal imaging technique for the ON\&LCN by reviewing imaging techniques used in studies of osteocyte anatomy, bone pathology and mechanobiology, including light microscopy (LM), electron microscopy (EM) and X-ray computed tomography (CT).

3. Examine the history and current state of the art in computational modelling of the ON\&LCN and discuss the importance of high-resolution 3D imaging to this field.

4. Delineate how future investigations on the ON\&LCN can be undertaken, taking into account recent progress in high-resolution 3D imaging.

Osteocyte imaging has been reviewed previously (Schneider et al., 2010; Webster et al., 2013; Kamioka, 2015). These reviews have covered 3D imaging techniques and high-resolution imaging, in vivo models, and the use of specialised imaging techniques for computational modelling. Investigations of the role of osteocytes in skeletal development has to date been limited. Osteocyte biology and pathology reviews include the work of Bonewald and Klein-Nulend and colleagues (Bonewald, 2013; Klein-Nulend et al., 2013), while computational modelling approaches have been reviewed in (Fritton and Weinbaum, 2009).

Here we take an integrated and holistic approach, reviewing together osteocyte anatomy, mechanobiology, imaging and computational modelling, their relationships to each other and to ongoing research in these different areas. This more complete and integrated review fits with the recent dominant discussion of the relevance of the Wnt signalling pathway in bone homeostasis and sclerostin as a therapeutic agent for osteoporosis (Niedzwiedzki and Filipowska, 2015). The state of the art in $3 \mathrm{D}$ imaging is brought up to date with the inclusion of improved and commercially available technologies for imaging. For instance, serial block-face scanning electron microscopy facilitates concurrent imaging of hard and soft tissues and thus has the potential to further enlighten the discussion of mechanobiology and pathology. 

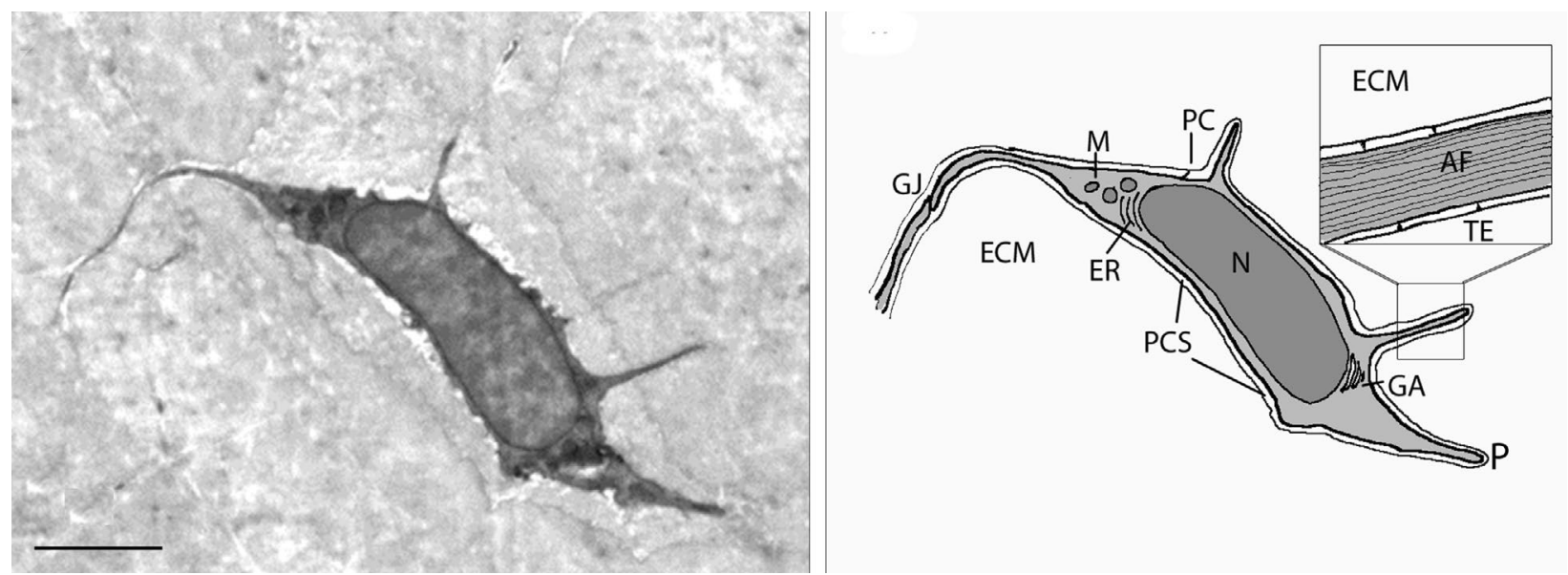

Fig. 2. ON\&LCN and typical cell machinery. (a) Transmission electron microscopy (TEM) image of murine bone (10-week-old C57BL/6 mouse, tibia). Bone tissue that is perfusion-fixed, decalcified, stained, and embedded in epoxy resin before thin-sectioning and imaging using TEM. Data acquired using a FEI Tecnai 12 TEM at $80 \mathrm{kV}$. Scale bar $=2 \mu \mathrm{m}$. (b) Diagram of the ON\&LCN and the typical cell machinery. Abbreviations: actin filaments (AF), endoplasmic reticulum (ER), extracellular matrix (ECM), gap junction (GJ), Golgi apparatus (GA), mitochondria $(\mathrm{M})$, nucleus $(\mathrm{N})$, osteocyte cell process $(\mathrm{P})$, primary cilium (PC), pericellular space (PCS), tethering elements (TE).

\section{Identification of microstructural phenotypes on cellular and sub-cellular scales of developing, ageing, healthy and diseased bone}

This section will review the microstructural bone phenotypes and specifically, the structures of the ON\&LCN for different bone conditions. For the purposes of this review the following nomenclature will be adopted to describe different hierarchical levels of bone structure: internal bone structure at cellular level, studied by light microscopy, including osteocyte cell bodies and osteocyte lacunae, internal bone microstructure at a level accessible by methods which overcome the diffraction limit of visible light, including osteocyte processes and canaliculi, and bone ultrastructure, including cell organelles, which can be observed by EM methods.

\section{Anatomy and physiology of the ON\&LCN}

Osteocytes are at once multi-functional communicators, mechanosensors and orchestrators of bone modelling and remodelling as well as regulators of calcium and phosphate homeostasis and endocrine cells. The role of the ON\&LCN as an important mechanosensory system of bone has been recognised over the last decade. Variations in the structure of the ON\&LCN, such as osteocyte/ lacunar density, number of processes/canaliculi per cell and lacunar porosity may have profound effects on bone mechanotransduction and mechanosensation.

\section{Microstructure of the $O N \& L C N$ and bone matrix}

Lacunar number density is reported to be between 20,000 and $37,000 / \mathrm{mm}^{3}$ in healthy tissue (Carter et al., 2013b; Dong et al., 2014). Osteocyte number density in remodelling bone is elevated compared with inactive tissue in both healthy controls and women with hip fracture. The 2-dimensional (2D) osteocyte density was found to be $647 / \mathrm{mm}^{2}$ in forming compared to $470 / \mathrm{mm}^{2}$ in quiescent tissue for fracture cases and $538 / \mathrm{mm}^{2}$ compared to $401 /$ $\mathrm{mm}^{2}$ in healthy controls, indicating that osteocytes may contribute to bone homeostasis (Power et al., 2002). Mice with targeted deletion of osteocytes are resistant to bone loss induced by unloading (Tatsumi et al., 2007), and load-related morphological and biochemical responses have been observed in osteocytes, which supports the notion that osteocytes act as mechanosensors (Zhang et al., 2006). Each osteocyte has 50-100 cell processes, that is about one million osteocytes or more per $\mathrm{mm}^{3}$ of bone tissue. Moreover, the shape and size of the osteocyte and the PCS reflect cell viability (Knothe Tate et al., 2004), while studies of rat bone have found a relationship between age and the number of canaliculi, (Okada et al., 2002). At the same time, the number and orientation of osteocyte cell processes are changed over the lifecourse (Holmbeck et al., 2005), with increased numbers of processes extending towards the vascularity than towards the mineralisation front when the cell is fully developed and enclosed in the mineralised matrix (Knothe Tate et al., 2004). Furthermore, time-lapse microscopy has been used to show that cultured osteocytes, observed during the mineralisation process, can expand and contract their cell body within their lacuna, and extend and retract their processes (Veno et al., 2007; Dallas and Bonewald, 2010). Thus, osteocytes could be considered as dynamic structures which may alter in response to external stimuli (Dallas et al., 2009; Dallas and Bonewald, 2010). Finally, changes to the shape, size and organisation of osteocytes have been observed in histological studies of pathological bone conditions (Knothe Tate et al., 2004), which will be discussed in more detail in the section regarding pathology.

It is also interesting to consider the effects of changes to the bone matrix surrounding the ON\&LCN. It has been shown that the amplification of strain in osteocyte lacunae is affected by the local bone tissue properties with maximum strain relating inversely to the perilacunar tissue modulus (Nicolella et al., 2006; Bonivtch et al., 2007). Therefore, changes in bone matrix properties will affect osteocyte mechanosensation. A recent study has revealed that the mass density of bone matrix varies with distance 
Fig. 3. Transverse cross-section of the idealised structural model for a cell process in a canaliculus attached to a focal attachment complex and tethered by the pericellular matrix. The sketch (not to scale) shows the osteocyte process and its attachment to the canalicular wall, including the structure of the central hexagonally-packed actin filament bundle and the arrangement of the fimbrin cross-bridges, cross-filaments, and transverse elements. Image from Wang et al. (2007) with kind permission of PNAS.

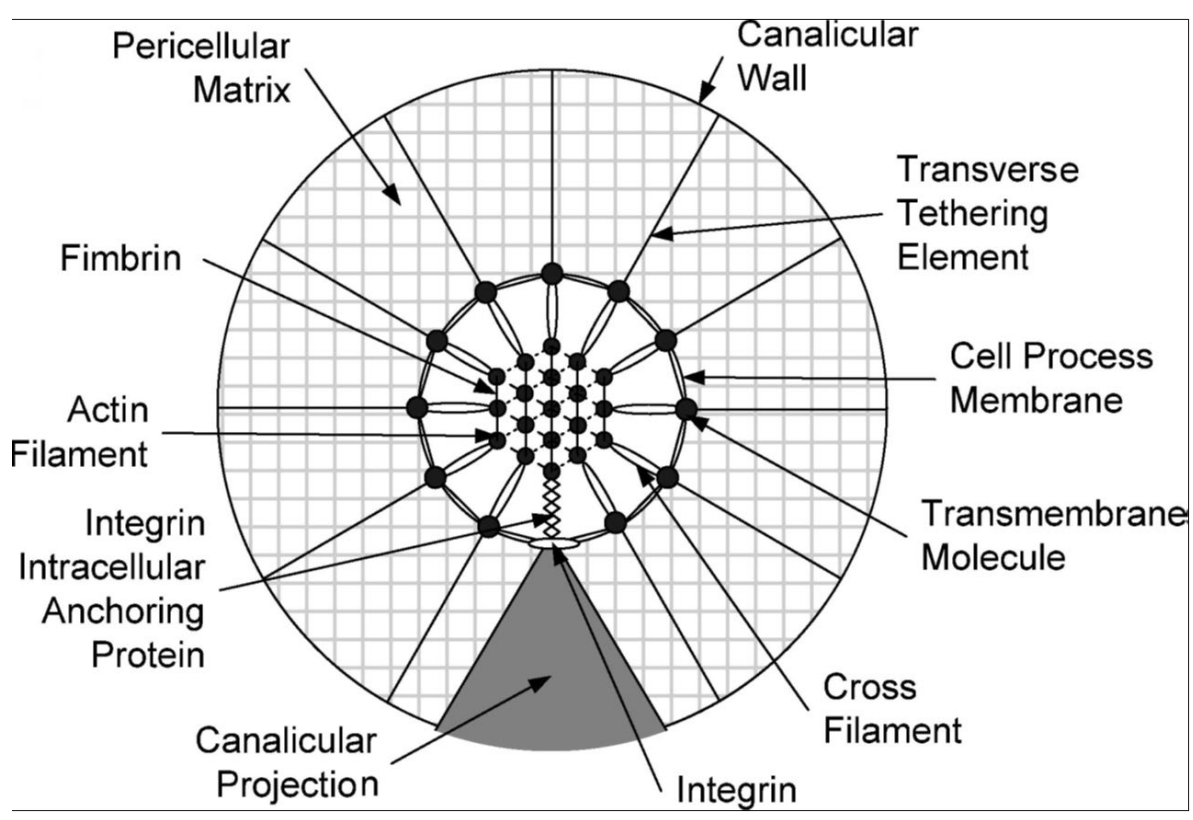

from the LCN (Hesse et al., 2015), with the matrix adjacent to the LCN porosity being most dense. This supports the hypothesis that mineral exchange occurs at the interface of the $\mathrm{LCN}$ in bone mineralisation and mineral homeostasis.

\section{ON\&LCN at the ultrastructural level}

In addition to the typical cell machinery (nucleus, mitochondria, endoplasmic reticulum, Golgi apparatus, etc.) the ON\&LCN exhibit other ultrastructural features important for mechanobiology, which are discussed in the following and illustrated in Fig. 2.

Tethering elements. Fluid flow within the LCN imposes shear stress, which deforms the osteocyte and its processes. A transmission electron microscopy (TEM) image-based model of strain amplification proposed by Han and colleagues (Han et al., 2004) introduced the concept of tethering elements, which structurally connect osteocyte processes and the surrounding mineralised canaliculi with proteins such as CD44, laminins and proteoglycans. The model predicts that fluid flow will deform the tethering elements, creating a drag force that then imposes a hoop strain on the actin bundles in the process. It has later been expanded to include integrin attachments to conical projections on the canalicular wall, which can amplify the mechanical strain (Fig.3) (Wang et al., 2007). Forces applied focally at integrin attachment sites on cultured osteocyte-like cells initiated intercellular signals in vivo (gap junction coupling and purinergic receptor signalling) on the cell processes but not on the cell body (Wu et al., 2013).

Glycocalyx. The strain amplification model described above for the tethering elements (Han et al., 2004; Wang et al., 2007) suggests that only the osteocyte processes are sensitive to mechanical load, and that the cell body is insensitive to strain. Studies on single osteocytes have shown that the processes are more mechanosensitive than the cell body (Adachi et al., 2009). However, studies using cultured cells, with stress applied experimentally, both to the osteocyte body and to the processes, suggest that both are mechanosensitive (Burra et al., 2010). Furthermore, these studies showed that the glycocalyx - a cell membrane coating, made up of glycoprotein and polysaccharide - of the osteocyte processes (but not of the cell body) plays an essential role for bone mechanotransduction (Burra $e t$ al., 2010). In particular, the glycocalyx of the osteocyte processes was found to be required for the formation of integrin attachments, which are possibly important mechanotransducers that convey mechanical signals to the osteocyte (Han et al., 2004; Burra et al., 2010). It has also been shown that an intact glycocalyx is necessary for certain biochemical signals to be released in response to fluid flow (Reilly et al., 2003).

Cytoskeleton. The cell cytoskeleton consists of a network of filaments in the cytoplasm, including actin, which gives the cell its shape and stability. Actin filaments are present in the cell body and form the core of osteocyte processes. The cytoskeleton is crucial for the maintenance of cell shape, membrane tension and cell-cell connections (Tanaka-Kamioka et al., 1998). The connection between actin filaments in the cell processes and the PCM is postulated to be important in strain transduction and amplification (You et al., 2001; Han et al., 2004; You et al., 2004).

Gap junctions and Connexin 43. Cells communicate by direct contact and exchange of small molecules through gap junctions formed by connexons that are aligned with those on neighbouring cells. Gap junctions have been found between osteocyte processes and between osteocytes and osteoblasts (Doty, 1981), raising the possibility that they can transmit information from signals sensed by embedded osteocytes to cells at the bone surface, for instance to regulate bone formation and resorption. Additionally, fluid flow has been shown to result in opening of gap junctions in osteocyte-like cells in culture (Cheng et al., 2001). It has been suggested that gap-junctional communication facilitates bone's response to load (Bonewald, 2011). However, other evidence suggests that inhibiting this communication can enhance bone formation during loading 
and preserve it during unloading, which is reviewed by Lloyd and colleagues (2014). Recent studies have shown that the osteocyte gap junction channel Connexin 43 plays an integral role in bone mechanobiology and osteocyte viability (Plotkin et al., 2015; Riquelme et al., 2015; Xu et al., 2015).

Primary cilia. Primary cilia are known to act as flow sensors in the embryo during left-right axis determination, and they are linked to mechanotransduction in polycystic kidney disease (Ong and Wheatley, 2003). In line with that, it has been suggested that osteocyte cilia may play a role in bone mechanotransduction and mechanosensation by acting as flow or strain sensors. It is postulated that primary cilia are involved in osteocyte mechanotransduction. Lee and colleagues have shown that fluid flow induces an increase in $\mathrm{Ca}^{2+}$ signalling (Lee et al., 2015). This hypothesis is supported by studies in mice, which show that deletion or disruption of ciliary genes leads to a disruption of mechanosensation (Xiao et al., 2011) or reduced bone formation (Qiu et al., 2012). Other studies have provided evidence that the deflection of primary cilia by fluid flow induces osteogenic and bone resorptive responses (Malone et al., 2007). However, different studies propose that cilia are only present on a small fraction of osteocytes and that they act as chemical rather than fluid flow sensors, whose function is related to mineralisation (Coughlin et al., 2015). These results conflict with studies carried out on rat tibiae, where $94 \%$ of osteocytes expressed primary cilia (Uzbekov et al., 2012), leaving the involvement of primary cilia in mechanotransduction open to debate.

Integrins. Integrins are receptors and transducers that connect the cytoskeleton and the extracellular matrix (ECM) as transmembrane cell adhesion proteins. It has been proposed that they play a role in bone mechanotransduction and mechanosensation (Salter et al., 1997; Haugh et al., 2015).

\section{Bone development}

The involvement of osteocytes in bone development has been little explored thus far. Endochondral ossification involves the differentiation of mesenchymal stem cells to chondrocytes, which form a cartilaginous matrix, which is subsequently mineralised and vascularised. The blood vessels bring in osteoblasts which bind to the cartilaginous matrix and deposit bone matrix. Some matrix-producing osteoblasts on the surface of bone embed in the osteoid matrix and become osteocytes. The embedding cell develops dendritic processes and changes from a polygonal to a stellate osteocyte, retaining connections with the surface and cells already embedded (Gilbert, 2000). It has been suggested that the embedding osteoid cell and the osteocyte play roles in the mineralisation process and mineral homeostasis (Mikuni-Takagaki et al., 1995; Teti and Zallone, 2009).

Publications on the involvement of osteocytes in developing bone and its relationship to ageing and health and disease are scarce but include a study of the development of craniofacial bone in zebrafish using confocal laser scanning microscopy (CLSM) and light sheet microscopy (LSM) (Jemielita et al., 2013), and further a study of the expression of sclerostin in the developing zebrafish using fluorescence microscopy (McNulty et al., 2012). Moreover, X-ray micro-computed tomography $(\mu \mathrm{CT})$ and histology have been used to study skeletal mineralisation in mice (Hafez et al., 2015). Finally, a study of rats at different ages, using acid-etched resin casting and scanning electron microscopy (SEM) to investigate the LCN, showed a developing canalicular system fusing with neighbouring canaliculi and round osteocyte lacunae with short and thick canaliculi (Okada et $a l ., 2002)$. For acid-etched resin casting, the LCN is filled by a resin and the mineralised bone matrix is etched away by acid so that the relief cast of the LCN can be imaged using SEM after coating with heavy metals (Feng et al., 2006). Investigation and imaging of developing bone phenotypes is an area which requires further exploration in order to clarify the functional role of osteocytes.

\section{Ageing, pathology and other bone states}

Various studies have compared ON\&LCN morphology, behaviour and changes in terms of age, gender, site, loading and pathology (summarised in Table 1), as outlined in the following.

\section{Age}

Lacunar number density decreases with age, by as much as 39 \% (Mullender et al., 1996; Qiu et al., 2002; Billings et al., 2012). A study using human bone tissue provided evidence that this decline is associated with the accumulation of microcracks (Vashishth et al., 2000). In the same study, Vashishth and colleagues noted that ageing bone tissue is characterised by increased heterogeneity in the spatial organisation of osteocytes. A 3D study of rat bone at different ages using resin casting of the LCN and subsequent SEM and CLSM demonstrated that the internal structure of compact bone changes with age (Okada et al., 2002). Mandibles of juvenile rats had round lacunae with short, thick canaliculi, while adults had flatter, more ellipsoidal lacunae with more canaliculi, which were branched to a greater extent. On the other hand, animals of advanced age had slender, flat lacunae with fewer, smaller canaliculi. Quantitative analysis of non-human primate bone using SEM provided evidence that lacunar density was decreased, lacunar cross-sectional area was increased and the number of canaliculi per lacuna remained the same (approx. 37) with age (Billings et al., 2012). Studies have found a relationship between age and both the number and orientation of osteocyte processes and canaliculi. A roughly $30 \%$ reduction in the number of canaliculi has been observed in elderly humans (Milovanovic et al., 2013). Conversely, Lai and co-workers have recently reported that the number density of canaliculi in mouse bone was unaffected by age, but that the dimensions of the PCS (in cortical but not in trabecular bone) increased due to an expansion of the canalicular wall (Lai et al., 2015).

\section{Gender}

Several studies suggest that there are no differences of the ON\&LCN related to gender in the non-osteoporotic population (Vashishth et al., 2000; Qiu et al., 2002; Jordan et al., 2003; Miszkiewicz, 2016). However, it has also been reported that osteocyte number density is $15 \%$ higher in 
Table 1. Anatomy of the osteocyte and the lacuno-canalicular network with ageing, pathology and other bone states.

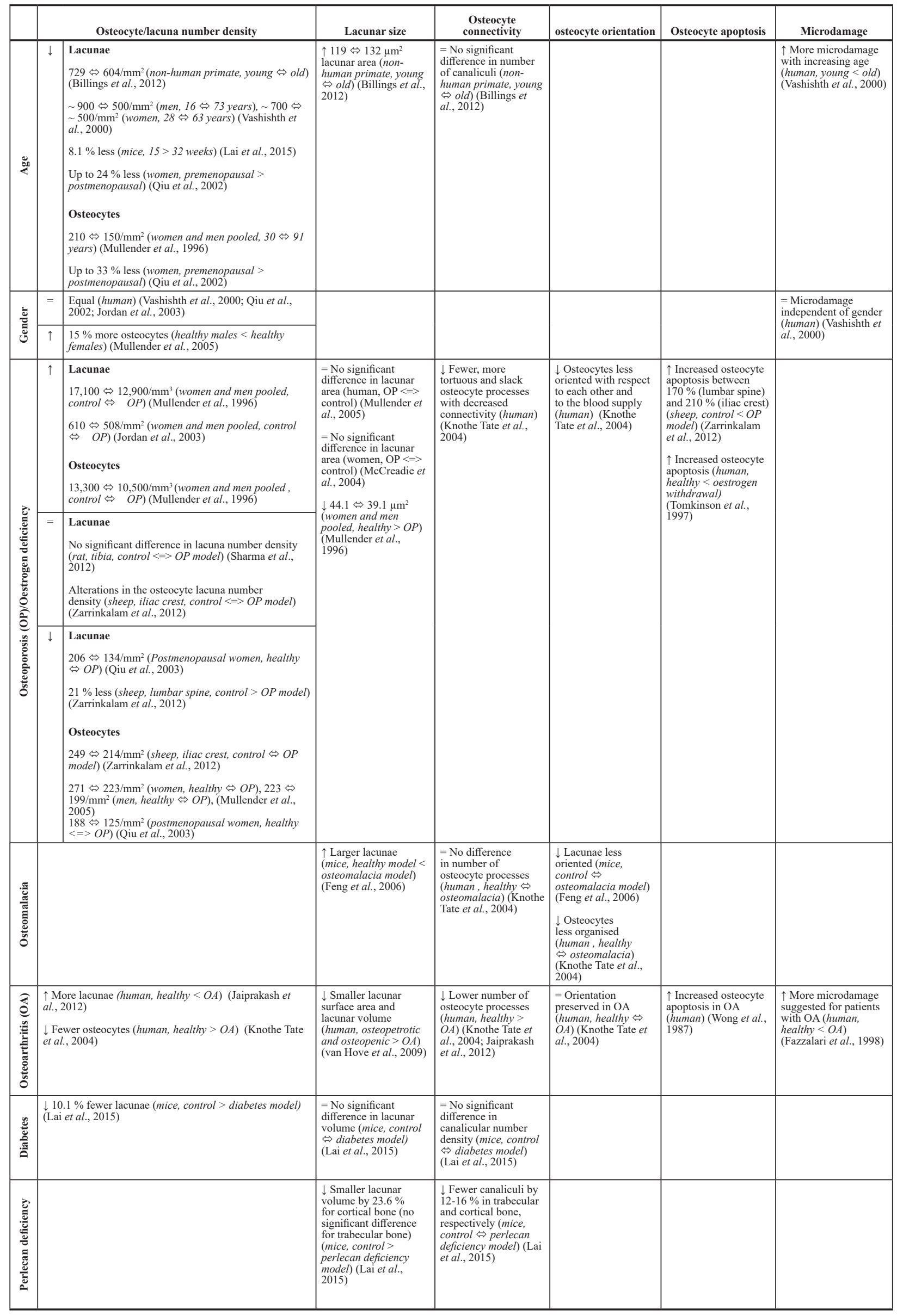


healthy females compared to healthy males (Mullender et al., 2005).

\section{Site/Loading}

Here, variations in osteocytes between bones from different sites are assumed to be due to the influence in loading direction and intensity and they are discussed together. Increased loading triggers the addition of new bone while unloading or disuse results in bone resorption. Various biochemical responses including sclerostin have been shown to be affected by loading of different frequency, timing and intensity, which are reviewed in (Bonewald, 2013).

Fibular osteocytes are reported to be elongated in shape and aligned with each other, parallel to the loading direction. On the other hand, calvarial osteocytes are more spherical or oblate and not aligned in any particular direction (Vatsa et al., 2008). A study of women aged from 20-86 years, where a reduction in loading with increasing age has been assumed, found that lacunae became smaller and more spherical (Carter et al., 2013a). In a synchrotron radiation-based CT (SR CT) study (Carter et al., 2014) it has been observed that lacunar number density of healthy human femora varied significantly within individuals, with the anterior region having a lower density than both medial and posterior regions. These variations are postulated to be related to loading and bending of the femoral shaft, but the reasons are unclear (Carter et al., 2014).

In summary, studies up to date suggest that lacunar number density decreases and lacunar area increases with age, but conflicting evidence exists regarding change in the numbers of canaliculi. With age, cell shape changes from round to slender and the size of the PCS increases, while the level of osteocyte organisation decreases. Studies on the influence of gender provide contradictory results, where most studies did not identify significant differences between males and females. Finally, osteocytes have been reported to align in the direction of loading and they are smaller when loading is reduced.

\section{Pathology}

Oestrogen deficiency and osteoporosis. The hormone oestrogen is essential for maintenance of the skeleton as it suppresses bone turnover and balances the formation and resorption of bone tissue, preventing bone loss (Cummings and Melton, 2002). Ovariectomy has been shown to increase bone turnover and induce bone loss (Wronski et al., 1985; Frost and Jee, 1992). Therefore, ovariectomised mice act as a model for osteoporosis, which mimics the reduction in oestrogen after the menopause, leading to an osteoporotic-like bone phenotype and related changes in bone structure and material properties, which are detrimental for bone mechanics (Frost and Jee, 1992). Studies have shown that ovariectomised rats have differences in bone matrix collagen arrangement, possibly changing the permeability of the matrix and affecting interstitial fluid flow (Sharma et al., 2012). Oestrogen deficiency has also been shown to induce apoptosis, which could alter osteocyte communication and interconnectedness (Tomkinson et al., 1997; Brennan et al., 2014a; Brennan et al., 2014b). Any or all of these changes could affect bone mechanics by modifying bone mechanotransduction pathways and with that, signals perceived by osteocytes from strain or fluid flow, eventually triggering osteocyte biochemical outputs.

A study demonstrated higher lacunar and osteocyte number densities in osteoporotic individuals and significantly reduced lacunar area in osteoporotic individuals (Mullender et al., 1996). Another study using human biopsies confirmed that osteocyte number density declines with age, where this decline occurs only in older bone and significantly in both pre- and post-menopausal women (Qiu et al., 2002).

Bone cells derived from patients with osteoporosis show an impaired osteocyte response to mechanical loading (Sterck et al., 1998). Osteoporotic patients have been reported to have $34 \%$ fewer osteocytes than healthy controls (Qiu et al., 2003). Overall, patients whose bone houses fewer osteocytes are more likely to sustain fracture, demonstrating their crucial role in bone maintenance (Qiu et al., 2003). To date, and to the best knowledge of the authors, it is not known yet if the lower osteocyte number is a cause or rather a consequence of osteoporosis.

The proportion of occupied lacunae was found to be the same in healthy and diseased patients, indicating that the change is not the result of premature osteocyte death, but a consequence of reduced osteocyte formation (Qiu et al., 2003). Using a sheep model for osteoporosis, reduced osteocyte number density, reduced bone volume, osteoid surface area and bone formation rate were observed, while the number density of empty lacunae increased, when compared to controls (Zarrinkalam et al., 2012). Another study found that osteocyte density was lower in osteoporotic females compared to healthy females (Mullender et al., 2005). A sample of osteoporotic bone exhibited a decrease in connectivity of the ON\&LCN compared to healthy bone and the orientation of osteocyte processes to each other and to the blood supply was decreased (Knothe Tate et al., 2002).

Osteoarthritis. Results based on acid-etched resin casting have shown that the osteocyte lacunar morphology is rougher and rounder in osteoarthritis compared to control samples, (Jaiprakash et al., 2012). A histological investigation showed an increase in the number of nonviable osteocytes in osteoarthritic patients compared to controls (Wong et al., 1987). A sample of osteoarthritic bone exhibited a decrease in connectivity of the ON\&LCN compared to healthy bone, while the orientation of osteocyte processes to each other and to the blood supply was preserved (Knothe Tate et al., 2002). Osteocytes in osteoarthritic human bone were found to be elongated and their lacunae smaller than those from osteopenic and osteopetrotic bone (van Hove et al., 2009).

Osteomalacia. Osteomalacia (known as rickets in children) is softening of bone due to hypomineralisation, through lack of vitamin D, or overactive resorption of calcium from the bone. The LCN in osteomalacia, characterised by an acid-etched resin casting study of mouse bone, has rough surfaces on the interior of the osteocyte lacunae and fewer canaliculi (Feng et al., 2006), where the surface roughness is most likely due to reduced and/ or uneven mineral content in the perilacunar matrix, which allows more resin to penetrate into the bone matrix. In ad- 
dition, the osteocyte lacunae in this study were larger and organised to a lesser extent than in control bone (Feng et al., 2006). In a sample of osteomalacic human bone the osteocytes appeared highly connected, but had tortuous processes (Knothe Tate et al., 2002, 2004). In addition, the ON observed in the same sample was found to be less organised ('chaotic') than in other pathological states.

Sclerostin-related bone diseases. Studies of sclerosteosis and van Buchem disease, both rare high-bone mass diseases caused by mutations in the SOST gene, have led to the identification of sclerostin as a key negative regulator of bone mass (Balemans et al., 2001; Staehling-Hampton et al., 2002). Sclerostin is a glycoprotein produced by osteocytes (and chondrocytes), which is encoded by the SOST gene. Sclerostin binds to LRP5/6 co-receptors and inhibits the intracellular Wnt signalling pathway, leading to decreased bone formation by osteoblasts, reviewed by Bonewald and Johnson as well as by Klein-Nulend and co-workers (Bonewald and Johnson, 2008; Klein-Nulend et $a l ., 2013)$. It is also suggested that sclerostin plays a role in the regulation of perilacunar mineralisation by osteocytes, enabling extraction of calcium from the perilacunar area when needed, followed by restoration of the mineralisation when the demand has been met (Atkins and Findlay, 2012; Kogawa et al., 2013).

Studies in rats found that treatment of osteoporotic animals with sclerostin antibody produced marked increases in bone formation, reversing the osteoporotic changes induced by ovariectomy (Li et al., 2009; Ominsky et al., 2014). In contrast to most other therapeutic treatments, which involve anti-resorptive agents and prevent or decelerate further bone loss, sclerostin has the potential to restore lost bone mass and strength. For example, two humanised sclerostin monoclonal antibodies have had positive effects on bone density, suggesting that neutralising sclerostin antibodies have potential as an osteoporosis treatment (Shah et al., 2015a). Phase III studies are ongoing in osteoporotic patients and results are awaited (Becker, 2014).

Osteonecrosis. Osteonecrosis is characterised by missing or dead osteocytes, where necrotic bone does not have the capacity to remodel. Osteonecrosis can be caused by alcohol abuse, radiation, sickle cell anaemia and also by treatment with glucocorticoids or bisphosphonates, reviewed in (Bonewald, 2013) and (Hesse et al., 2014). The mechanisms of osteonecrosis are unclear but may be linked to osteocyte apoptosis (Calder et al., 2004).

Diabetes. Impaired osteoblast differentiation and activity are associated with diabetes and Type 1 diabetic osteoporosis (Motyl et al., 2012). In another study CLSM and TEM has been used to examine quantitatively the topology and structure of the ON\&LCN in diabetic mice (Lai et al., 2015). The results for lacunar volume, lacunar spacing, canalicular number density, PCS dimensions and canalicular space dimensions were similar to healthy animals; however, the lacunar number density was reduced approximately $10 \%$, probably linked to osteoblast differentiation impairment. The canalicular wall area and cell process area were significantly reduced in diabetic cortical bone.
Perlecan deficiency. (Human Schwartz-Jampel syndrome) Perlecan is an essential component of the PCM that maintains an adequate fluid/solute transport pathway by possibly inhibiting mineralisation of the PCS (Farach-Carson and Carson, 2007). An investigation of the ON\&LCN morphology in perlecan-deficient mice showed that the dimensions of the pericellular area between the osteocyte process and the canalicular wall decrreased (Thompson et al., 2011).

\section{Imaging of the ON\&LCN}

Current techniques to image the ON\&LCN include LM, TEM and SEM, CLSM, CT, transmission X-ray microscopy (TXM), ptychographic X-ray CT, X-ray phase tomography (holotomography)/X-ray phase nanotomography, serial focused ion beam SEM (serial FIB SEM) and atomic force microscopy (AFM). For a summary of the various imaging techniques discussed, relevant references and their advantages and disadvantages please see Table 2 .

\section{The ideal imaging method}

2D images provide only a small part of the complete context of any cell. Accurate morphology including cell shape, size or location cannot be derived reliably from 2D images. Furthermore, the relationship between structures both within and without a cell - are often invisible in 2D. Viewing and analysing structures in a 3D context avoids misinterpretation. It is however a challenge to obtain $3 \mathrm{D}$ data, especially at high spatial resolutions. Novel (highresolution) 3D imaging techniques are currently being developed as discussed at the end of this section on imaging of the ON\&LCN and at the end of this review on the future role of high-resolution 3D imaging in osteocyte anatomy and pathology.

In addition to providing 3D data, the ideal technique for imaging bone cells and their ECM at cellular and subcellular scales would be a high-resolution, non-destructive method, capable of imaging large volumes in situ and in vivo and offering concurrent hard and soft tissue image contrast. Furthermore, the imaging technique(s) of choice would be widely available at a reasonable cost and produce quantitative hallmarks of healthy and diseased tissue, which are easy to interpret and analyse.

To meet all of these criteria compromises have to be made in practice. The ON\&LCN extend throughout the bone matrix. To reveal the true degree of connectedness between contiguous osteocytes the ideal imaging method should be able to visualise a volume containing many cells. However, in the real world, different imaging techniques depend on various sample preparation protocols and there exists a broad range of experimental limitations during imaging. Most imaging techniques require tissue to be excised from the body, many require mechanical sectioning to some degree, and some of the techniques need harsh chemical treatment to render the tissue suitable for imaging, which can change the state of the native biological sample and thus, complicate interpretation of the gathered image data (Weston et al., 2010). 
Table 2. Imaging techniques for the assessment of the osteocyte and lacuno-canalicular network.

\begin{tabular}{|c|c|c|c|c|c|c|}
\hline Technique & 2D/3D & Resolution & $\begin{array}{c}\text { Typical } \\
\text { volume * }\end{array}$ & Destructive? & Comments & Reference \\
\hline \multicolumn{7}{|c|}{ LIGHT MICROSCOPY } \\
\hline light microscopy (LM) & $2 \mathrm{D}$ & $200 \mathrm{~nm}$ & - & No & $\begin{array}{l}\text { - Functional imaging through } \\
\text { fluorescent labelling } \\
\text { - Soft tissue contrast }\end{array}$ & Marotti et al., 1995 \\
\hline $\begin{array}{l}\text { High-resolution episcopic } \\
\text { microscopy }\end{array}$ & $3 \mathrm{D}$ & $1 \mu \mathrm{m}$ & $>1 \mathrm{~cm}^{3}$ & No & - Specific labelling possible & $\begin{array}{l}\text { Mohun and Weninger, } \\
2012\end{array}$ \\
\hline Light sheet microscopy & $3 \mathrm{D}$ & $10 \mu \mathrm{m}$ & $>0.5 \mathrm{~cm}^{3}$ & No & $\begin{array}{l}\text { - Live cell imaging possible } \\
\text { - Requires optical clearing }\end{array}$ & Weber et al., 2014 \\
\hline $\begin{array}{l}\text { Confocal laser scanning } \\
\text { microscopy (CLSM) }\end{array}$ & $3 \mathrm{D}$ & $200 \mathrm{~nm}$ & $0.1 \mathrm{~mm}^{3}$ & No & $\begin{array}{l}\text { - Functional imaging through } \\
\text { fluorescent labelling } \\
\text { - Soft tissue contrast } \\
\text { - Limited range of depth }\end{array}$ & Kamioka et al., 2001 \\
\hline \multicolumn{7}{|c|}{ ELECTRON MICROSCOPY } \\
\hline $\begin{array}{l}\text { Scanning electron } \\
\text { microscopy (SEM) }\end{array}$ & $2 \mathrm{D}$ & $3 \mathrm{~nm}$ & - & No & - Inherently 2D & Okada et al., 2002 \\
\hline $\begin{array}{l}\text { Serial section SEM/ } \\
\text { automated tape-collecting } \\
\text { ultramicrotome (ATUM) } \\
\text { SEM }\end{array}$ & $3 \mathrm{D}$ & $\begin{array}{l}2 \mathrm{~nm}(x / y) \\
\text { and } 60- \\
90 \mathrm{~nm}(z)\end{array}$ & $10^{9} \mu \mathrm{m}^{3}$ & No & $\begin{array}{l}\text { - Complex sample } \\
\text { preparation }\end{array}$ & Tapia et al., 2012 \\
\hline $\begin{array}{l}\text { Transmission electron } \\
\text { microscopy (TEM) }\end{array}$ & $2 \mathrm{D}$ & $<1 \mathrm{~nm}$ & - & No & $\begin{array}{l}\text { - Limited field of view } \\
\text { - Complex sample } \\
\text { preparation }\end{array}$ & You et al., 2004 \\
\hline $\begin{array}{l}\text { TEM tomography and } \\
\text { serial TEM tomography }\end{array}$ & $3 \mathrm{D}$ & $2 \mathrm{~nm}$ & $>10 \mu \mathrm{m}^{3}$ & No & $\begin{array}{l}\text { - Long acquisition times } \\
\text { - Missing wedge problem }\end{array}$ & $\begin{array}{l}\text { Bonetta, 2005; Kamioka } \\
\text { et al., } 2009\end{array}$ \\
\hline Serial section TEM & $3 \mathrm{D}$ & $\begin{array}{l}<1 \mathrm{~nm} \\
(x / y) \text { and } \\
60 \mathrm{~nm}(z)\end{array}$ & $>10^{3} \mu \mathrm{m}^{3}$ & No & $\begin{array}{l}\text { - Technically difficult } \\
\text { - Time-consuming }\end{array}$ & $\begin{array}{l}\text { Harris et al., 2006; } \\
\text { Palumbo et al., } 1990\end{array}$ \\
\hline $\begin{array}{l}\text { Serial focused ion beam } \\
\text { SEM (serial FIB SEM) }\end{array}$ & $3 \mathrm{D}$ & $<3 \mathrm{~nm}$ & $10^{4} \mu \mathrm{m}^{3}$ & Yes & $\begin{array}{l}\text { - Slow } \\
\text { - field of view }\end{array}$ & Schneider et al., 2011 \\
\hline $\begin{array}{l}\text { Serial block-face SEM } \\
\text { (SBF SEM) }\end{array}$ & $3 \mathrm{D}$ & $<10 \mathrm{~nm}$ & $10^{5} \mu \mathrm{m}^{3}$ & Yes & $\begin{array}{l}\text { - Extensive sample } \\
\text { preparation }\end{array}$ & $\begin{array}{l}\text { Peddie and Collinson, } \\
2014\end{array}$ \\
\hline \multicolumn{7}{|c|}{ X-RAY TECHNIQUES } \\
\hline $\begin{array}{l}\text { X-ray absorption CT } \\
\text { (lab-based } \mu \text { CT and } \\
\text { synchrotron radiation- } \\
\text { based CT) } \\
\end{array}$ & $3 \mathrm{D}$ & $<1 \mu \mathrm{m}$ & $>10^{9} \mu \mathrm{m}^{3}$ & No & $\begin{array}{l}\text { - High radiation dose } \\
\text { - Simple sample preparation } \\
\text { - Relative sub- } \mu \text { m mineral } \\
\text { density quantification } \\
\end{array}$ & (Schneider et al., 2007) \\
\hline Ptychographic X-ray CT & $3 \mathrm{D}$ & $<100 \mathrm{~nm}$ & $1.7 \times 10^{3} \mu \mathrm{m}^{3}$ & No & $\begin{array}{l}\text { High radiation dose } \\
\text { - Absolute sub- } \mu \mathrm{m} \text { mineral } \\
\text { density quantification }\end{array}$ & (Dierolf et al., 2010) \\
\hline $\begin{array}{l}\text { Transmission X-ray } \\
\text { microscopy (TXM) CT }\end{array}$ & $3 \mathrm{D}$ & $<50 \mathrm{~nm}$ & $4.5 \times 10^{3} \mu \mathrm{m}^{3}$ & No & $\begin{array}{l}\text { - Chemical information } \\
\text { - High radiation dose } \\
\text { - Relative sub- } \mu \text { m mineral } \\
\text { density quantification }\end{array}$ & (Andrews et al., 2010) \\
\hline $\begin{array}{l}\text { X-ray phase tomography } \\
\text { (holotomography)/X-ray } \\
\text { phase nanotomography }\end{array}$ & $3 \mathrm{D}$ & $\begin{array}{c}120- \\
150 \mathrm{~nm}\end{array}$ & $>10^{9} \mu \mathrm{m}^{3}$ & No & $\begin{array}{l}\text { - Requires access to } \\
\text { synchrotron radiation } \\
\text { facility } \\
\text { - Simple sample preparation } \\
\end{array}$ & (Langer et al., 2012) \\
\hline \multicolumn{7}{|c|}{ SCANNING PROBE MICROSCOPY } \\
\hline $\begin{array}{l}\text { Atomic force microscopy } \\
\text { (AFM) }\end{array}$ & $2 \mathrm{D}$ & $20-50 \mathrm{~nm}$ & - & No & $\begin{array}{l}\text { - Information on mechanical } \\
\text { properties } \\
\text { - Imaging in aqueous } \\
\text { medium possible } \\
\end{array}$ & (Reilly et al., 2001) \\
\hline
\end{tabular}

* without tiling.

Many imaging modalities have been used in highresolution studies of the ON\&LCN. Each has advantages and disadvantages. For comprehensive comparisons of these techniques see (Schneider et al., 2011), Fig. 4, Table 2 and the following sections. In brief, LM, including CLSM, allows functional imaging of cells but is limited in spatial resolution by the diffraction limit of visible light $(\sim 200 \mathrm{~nm})$. EM provides high-resolution images of cell ultrastructure, including the organelles, cytoskeletal components and membranes, but requires complex sample preparation. In addition, EM is an inherently two-dimensional (2D) technique. In contrast, X-ray CT has enabled timely imaging of large volumes of bone tissue without the need for complex sample preparation. Standard X-ray imaging techniques however lack sufficient resolution for substantial sub-micrometre imaging and do not provide enough imaging contrast for weakly X-ray absorbing soft tissues such as the ON. This limitation for soft tissues means that using standard (i.e., absorptionbased) X-ray imaging, only the hard mineralised bone matrix - the negative imprint of the $\mathrm{ON}$ - can be assessed at sufficiently high contrast levels, but not the weakly X-ray- 


\section{Resolution}

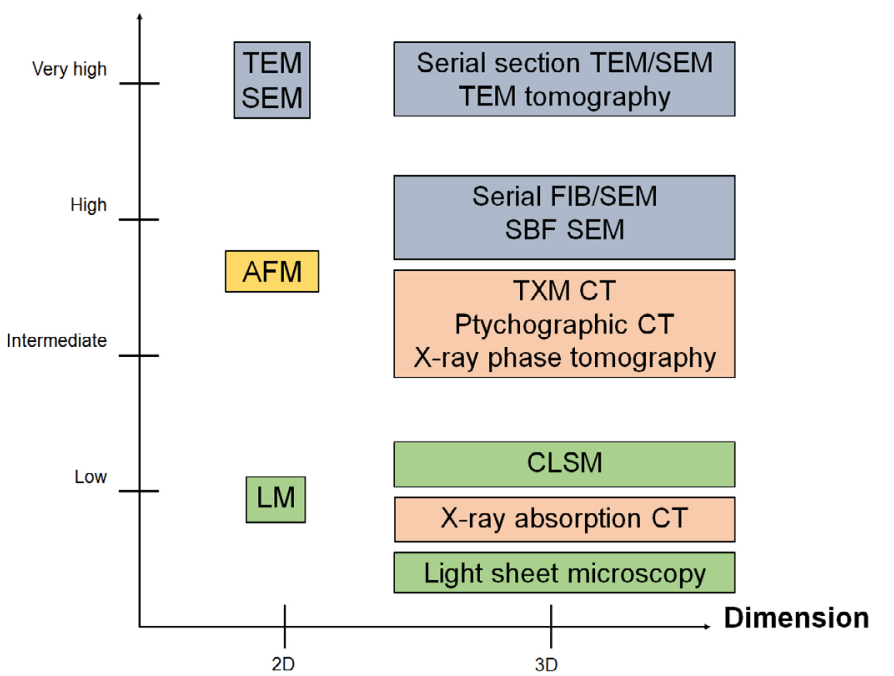

\begin{tabular}{l|}
\hline LIGHT MICROSCOPY \\
\hline ELECTRON MICROSCOPY \\
\hline X-RAY TECHNIQUES \\
\hline SCANNING PROBE MICROSCOPY \\
\hline
\end{tabular}

Fig. 4. Summary of imaging techniques for the assessment of the ON\&LCN. The various techniques are grouped regarding their dimensionality (2D or 3D) and currently achievable spatial resolution. Please see Table 2 for further details regarding the individual techniques. Abbreviations: Atomic force microscopy (AFM), confocal laser scanning microscopy (CLSM), light microscopy (LM), scanning electron microscopy (SEM), serial block-face SEM (SBF SEM), serial focused ion beam SEM (serial FIB SEM), transmission electron microscopy (TEM), transmission X-ray microscopy CT (TXM CT). Figure adapted from (Schneider et al., 2011) with kind permission of Elsevier.

absorbing soft cells within the LCN. This weak vision for soft tissues reduces the informative value of such results when used for instance in computational modelling studies aimed at understanding mechanotransduction processes using cell geometries based on experimental data.

When evaluating imaging modalities used for the assessment of biological tissue, and particularly for the ON\&LCN, there are many factors to consider, which are discussed in the following.

\section{Sample preparation and method validation}

Imaging tissue in its native state is an often elusive ideal. Chemical interventions to preserve tissue and/or enhance image quality, such as heavy metal staining or decalcification, can have unwanted effects such as tissue shrinkage (Buytaert et al., 2014). Where artefacts are introduced the microscopist must be aware of their impact and be able to compensate for them experimentally or post hoc during data processing. Nanophosphor particles delivered by bioballistic particle delivery systems can be used to monitor morphological changes in tissue during processing (Bushong et al., 2015). As bone is a composite material made up of a hard mineralised and a soft collagenous phase, the task of measuring artefactual change and of identifying and applying validating techniques, in order to ensure that the native tissue state is preserved and assessed, is more challenging than in homogenous tissues. Novel fixation techniques have been applied to osteocytes to optimise preservation. Acrolein, a small molecule which penetrates the tissue rapidly, has been used as fixative to improve the cell membrane preservation and reduce shrinkage (McNamara et al., 2009). Rutheniumbased fixatives and stains have also been investigated and found to enhance the preservation of cell and matrix proteoglycans (You et al., 2004; Shah et al., 2015b).

Validation of sample preparation and imaging techniques, to make sure that results accurately reflect the tissues and cells being examined, is important. Using more than one technique on the same sample and comparing the results can increase confidence in a novel method (Smith et al., 2006). Comparisons between the same tissues prepared for 'standard' EM and using cryo methods provide insight into changes which occur during sample preparation. Statistical methods have been used to overcome limitations of some EM data (Russo and Passmore, 2014). Beyond that, the issue of validating new 3D EM techniques has been reviewed by a task force set up to provide recommendations in 2012 (Henderson et al., 2012). It resulted in the creation of a unified data resource called EMDataBank (http://www. emdatabank.org), with the aim to develop validation reports and establish validation standards for 3D EM.

Adequate spatial resolution of microstructural and ultrastructural detail

Imaging techniques are needed that can resolve the detail of the cell processes (diameters in the order of $\sim 100 \mathrm{~nm}$ ) and ultrastructural cell details (You et al., 2004).

Adequate volume to visualise cell networks and tissue matrix

For most imaging techniques there exists an inverse relationship between spatial resolution and maximum possible sample volume to be assessed, due to experimental limitations. These limitations can be found on the side of image acquisition, such as the dimension of the detector or the time available to record images at a sufficient 
Fig. 5. Light microscopy (LM). A reflected polarised light micrograph of a secondary osteon in human tibia showing only the canaliculi lying in the light-reflecting plane. From (Marotti et al., 1995) with kind permission from Elsevier. (Editor's note: Normally, eCM Journal insists on such figures showing a meaningful scale bar. As this figure is a reproduction of a previously published figure, this was not possible)

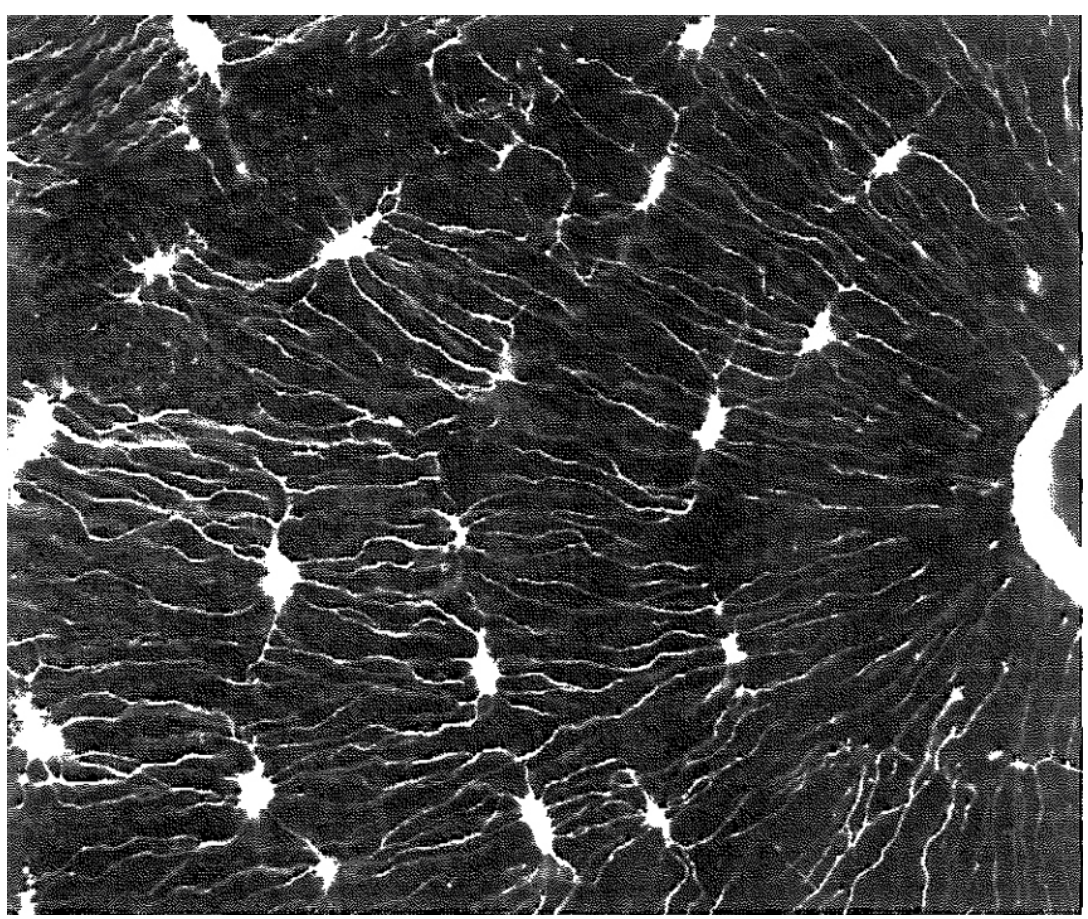

signal-to-noise ratio. Consequently, sampling smaller volumes allows working at higher spatial resolutions, while mitigating to some extent the problem of the limited penetration depth of the light and electrons used for imaging. However, small volume analysis introduces sampling errors and reduces the validity of results as the smaller number of cells examined cannot be considered to be representative (anymore) of the tissue. Due to the high tissue penetration depth of hard X-rays (energies typically $>10 \mathrm{keV}$ ), X-ray CT offers the largest volumes of interest of all the imaging techniques under consideration here, with whole-bone scanning being common. The volume of tissue which may be analysed using other techniques can also be limited by physical conditions such as the technical challenges of producing serial TEM sections, the restriction of the field of view in serial FIB SEM to sometimes less than $1000 \mu \mathrm{m}^{2}$ (Knott and Genoud, 2013; Peddie and Collinson, 2014) and the loss of the 'missing wedge' in electron tomography (Ercius et al., 2015).

\section{$3 D$ visualistation}

Elucidation of the mechanisms of mechanotransduction will require images of the ON\&LCN in $3 \mathrm{D}$ at different levels (cellular, sub-cellular, ultrastructural). LM and CT techniques produce 3D data at low $(\sim 300 \mathrm{~nm})$ and high $(<100 \mathrm{~nm})$ spatial resolutions. While traditional EM techniques are $2 \mathrm{D}$, the relatively new techniques of serial FIB SEM and serial block-face SEM (SBF SEM), produce 3D data sets by repeatedly imaging a resin block-face, as nanoscopic tissue sections are removed from the surface, either by an ion beam (serial FIB SEM) or by a remotely controlled ultramicrotome inside an SEM (SBF SEM) (Peddie and Collinson, 2014).

\section{Light microscopy}

'Standard' light microscopy (LM)

Since Hooke first observed and coined the term 'cell' in 1665 , light microscopy (LM) has succeeded in elucidating details of the structure and function of bone tissue (Fig. 5). Much work has been carried out using LM on stained histological bone sections, fundamentally a 2D observation method (Marotti et al., 1995; Remaggi et al., 1998; Ferretti et al., 1999; Hirose et al., 2007). Improved optics and the advent of digital imaging have enhanced the capabilities of LM, but as with all light-based microscopy methods, the spatial resolution is restricted by the diffraction limit of visible light to around $200 \mathrm{~nm}$. Additionally, as light penetration is limited in calcified tissue, decalcification may be necessary to optimise LM results. The spatial resolution of LM is insufficient to image the ON\&LCN completely, as the cell process diameters are around $100 \mathrm{~nm}$ (You et al., 2004). Nevertheless, specialised techniques such as reflected polarised LM have been used to quantify canaliculi (Marotti et al., 1995). Highresolution episcopic microscopy is a technique designed to provide $3 \mathrm{D}$ histological information by consecutive imaging of a wax block as it is sectioned (Mohun and Weninger, 2012), which has not been applied to image the ON\&LCN to date.

\section{Confocal laser scanning microscopy (CLSM)}

In confocal laser scanning microscopy (CLSM) a point source laser light excites tissue which is either autofluorescent (e.g. bone matrix through high level of collagen content) or has been stained with fluorescent dyes specific for cell components (e.g. actin filaments stained with phalloidin). Tissue is imaged at sequential focal planes using a pinhole detector to exclude light outside of the focal plane. A stack of 2D optical sections is acquired, which enables production of 3D representations of internal 
Fig. 6. Confocal laser scanning microscopy (CLSM). A fluorescence image of a chick calvarial fragment reconstructed from 40 confocal images. Cells labelled in green are osteocytes, and cells in red are osteoblasts on the surface of the bone. The staining clearly delineates the osteocyte processes. Scale bar $=20 \mu \mathrm{m}$. Adapted from (Kamioka et al., 2001) with kind permission of Elsevier.

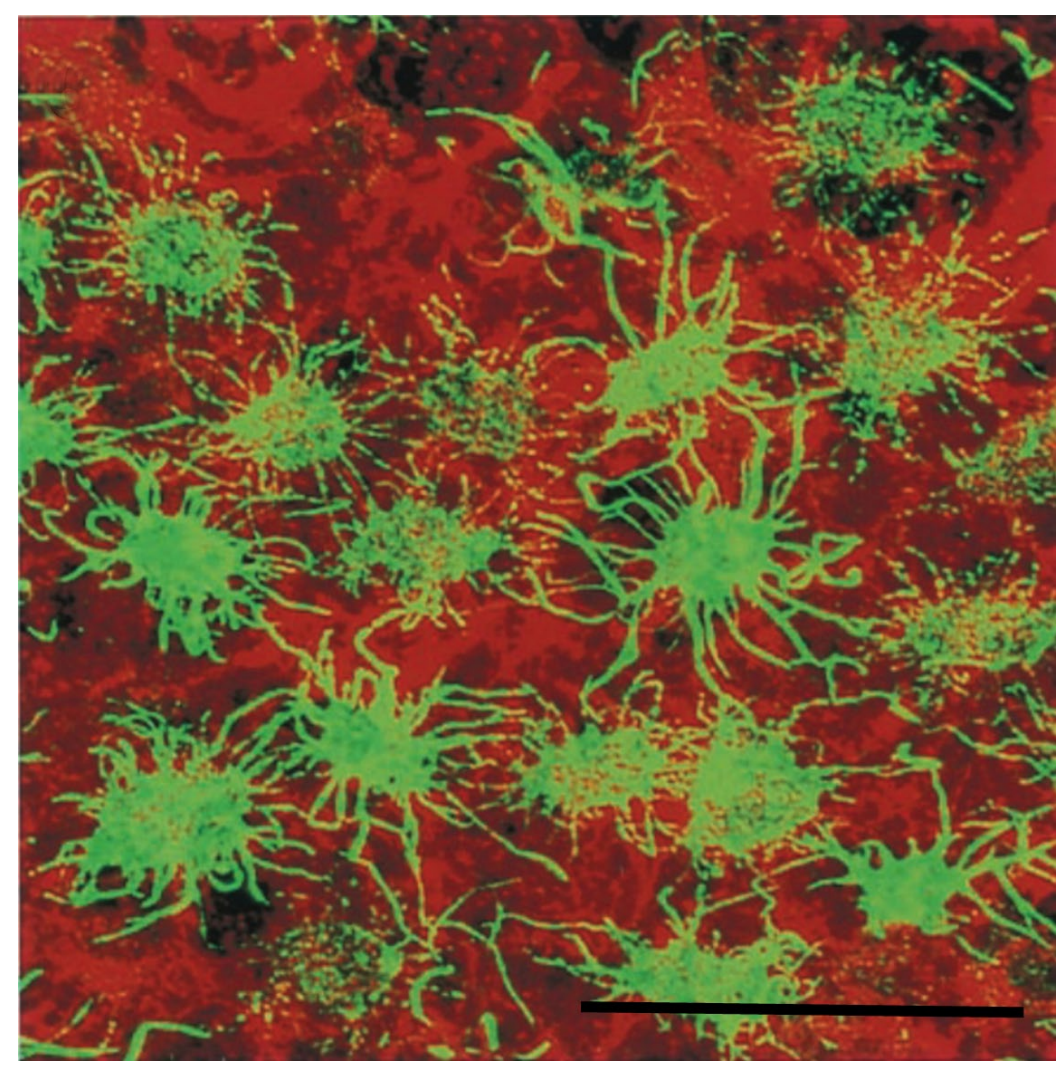

bone microstructures (Fig. 6). CLSM images have a spatial resolution of at best $200 \mathrm{~nm}$ in-plane and slightly worse (up to $450 \mathrm{~nm}$ ) out-of-plane, dependent on the objective lens employed. As in standard LM, this is not sufficient to image the ON\&LCN structure fully as the diameter of the osteocyte processes is in the order of $100 \mathrm{~nm}$ (You et al., 2004). The limitations of CLSM, when imaging in dense mineralised tissue, include the limited working distance (90-300 $\mu \mathrm{m})$ of high-quality, high-numerical aperture objectives and the decrease in signal with depth from light absorption and scattering. The practically achievable penetration depth of CLSM in mineralised tissue is 100$150 \mu \mathrm{m}$ (Jones et al., 2005). The difference in resolution in the $x / y$-direction and the $z$-direction can also make 3D reconstructions, quantification and interpretation of data more challenging. Finally, CLSM requires tissue to be cut, processed and cleared, thus moving it away from its native state. CLSM has been successfully used to characterise the spatial organisation, orientation and morphology of the ON\&LCN (Kamioka et al., 2001; Vatsa et al., 2008; Sugawara et al., 2011; Cardoso et al., 2013; KamelElSayed et al., 2015).

\section{Live cell imaging}

Time-lapse LM is used to observe and capture cellular dynamics by imaging live cells at predetermined regular time intervals in bright-field, phase-contrast and CLSM systems. A camera captures sequences of images which are later viewed at faster speed. Live cell imaging has shown that the osteocyte embedding process in living bone tissue is highly dynamic and that the cells can extend and retract their dendrites, making temporary connections with already embedded osteocytes (Dallas et al., 2009; Dallas and Bonewald, 2010). In studies of unloaded rat bone with the tracers procion red and microperoxidase diffusion transport mechanisms have been examined (Knothe Tate et al., 1998). Tracer injections have also been used to demonstrate the connection between the blood and canalicular fluid (Wang et al., 2004).

\section{Light sheet microscopy (LSM)}

In light sheet microscopy (LSM) a sheet of laser light is used in place of the point source in CLSM (Weber et al., 2014). Tissue is illuminated and imaged in slices as the laser scans through the sample. LSM provides images of intermediate spatial resolution $(\sim 10 \mu \mathrm{m})$ from optically cleared samples up to $1 \mathrm{~cm}^{3}$ in size. LSM has so far not been used extensively for bone imaging. Studies to date include the development of bone in zebrafish embryos (Jemielita et al., 2013) and simultaneous imaging of the soft and hard tissue of mouse cochlea (Buytaert et al., 2013). The reduced amount of photodamage induced and the ability to image larger volumes than for CLSM are both advantages of LSM, which should lead to LSM being more widely used in future osteocyte studies.

\section{Electron microscopy}

Electron microscopy (EM) takes advantage of the shorter wavelength of electrons compared to visible light to extend resolving power. EM uses a beam of accelerated electrons to improve the theoretical spatial resolution to the atomic range with aberration corrected instruments (Pennycook et al., 2015). In practice, the best spatial resolution for biological tissue is approximately $2 \mathrm{~nm}$. 


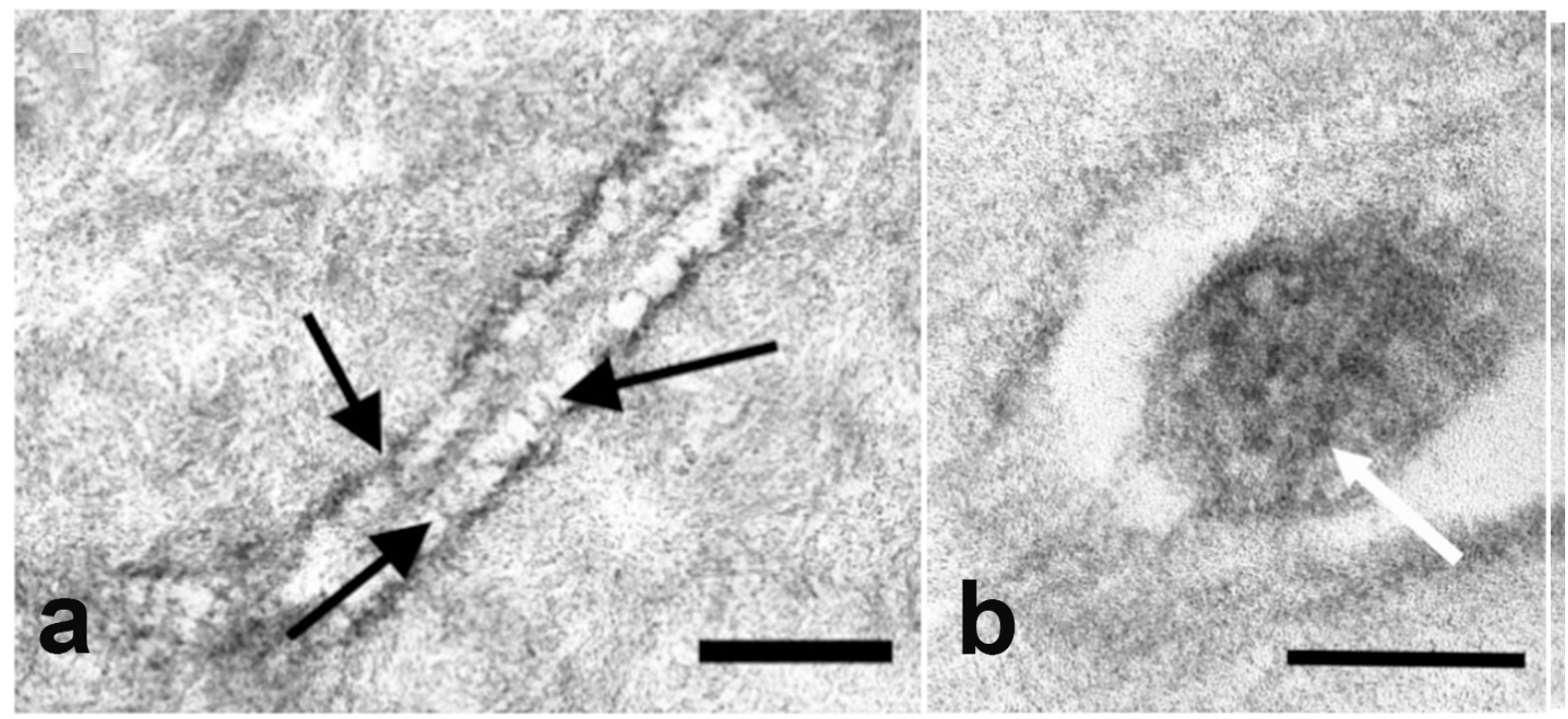

Fig. 7. Transmission electron microscopy (TEM) (a) TEM image showing an osteocyte process in longitudinal section. Transverse elements (arrows) are shown connecting the cell process to the matrix. Scale bar $=300 \mathrm{~nm}$. (b) TEM image showing an osteocyte process in a transverse section. Dark spots (arrow) are cytoskeletal filaments, likely actin, sectioned transversally. Scale bar $=100 \mathrm{~nm}$. From (You et al., 2004) with kind permission of John Wiley \& Sons.

Transmission electron microscopy (TEM)

In transmission electron microscopy (TEM) electromagnetic lenses focus a beam of electrons which is transmitted through an ultrathin $(\leq 100 \mathrm{~nm})$ section of fixed, stained, dehydrated and resin-embedded tissue, where some electrons pass through the tissue and others are scattered to form an image. This has been the favoured technique for high-resolution imaging of cell ultrastructure since its development in the 1930s. For TEM, tissue must be prepared as ultrathin sections capable of withstanding low pressure (i.e. high vacuum) and bombardment by highenergy electrons. This requires involved sample processing using chemicals and heat that can introduce artefacts such as shrinkage. Bone presents the further challenge of a hard, mineralised matrix requiring an additional step of decalcification to facilitate sectioning (An and Martin, 2003). Cryofixation is known to preserve tissue in a more life-like state but has the disadvantage that only small $(\sim 200 \mu \mathrm{m}$ deep) volumes of tissue can be preserved. The first published record of TEM bone studies dates back to 1950 (Barbour, 1950). More recently it has been used to investigate tethering fibres between osteocytes and the PCM on a nanoscopic scale (Fig. 7) (You et al., 2004; McNamara et al., 2009) and the organisation of collagen and crystals in the bone matrix (Rubin and Jasiuk, 2005).

The 2D nature of TEM imaging has been somewhat overcome by the introduction of serial section TEM, TEM tomography and ultra-high voltage EM (UHVEM). Serial section TEM is technically difficult and time-consuming but provides high-resolution results (White et al., 1986; Harris et al., 2006) such as those used to elucidate the 3D shape of osteocytes (Palumbo et al., 1990). TEM tomography involves imaging a section repeatedly while it is incrementally tilted around its axis (Bonetta, 2005; McIntosh et al., 2005). The series of projections is used to reconstruct a 3D image of the sample. One disadvantage is the 'missing wedge' caused by the limited tilt range of about $60^{\circ}$ in most TEMs. UHVEM is not widely available and can only be used to image small samples (typically 3-5 $\mu \mathrm{m}$ sections (Takaoka et al., 2008)) due to the substantially limited penetration depth of electrons in hard tissues.

Nevertheless, UHVEM studies (Fig. 8) have produced $3 \mathrm{D}$ images of osteocyte processes at $16 \mathrm{~nm}$ resolution (Kamioka et al., 2009) and portions of the canaliculi down to around $2 \mathrm{~nm}$ resolution, providing significant information which contradicts previous studies (Kamioka et al., 2012). This is discussed further in the section of this review entitled 'The importance of high-resolution 3D imaging to computational modelling in bone mechanobiology'.

\section{Scanning electron microscopy (SEM)}

Scanning electron microscopy (SEM) employs a beam of electrons which is raster-scanned over a sample, usually fixed, dehydrated, dried and made conductive with a metal coating. The incident beam interacts with the sample producing secondary electrons, backscattered electrons and $\mathrm{X}$-rays (among other signals) that are collected to form an image. SEM resolution, which can range from about $20 \mu \mathrm{m}$ to $0.4 \mathrm{~nm}$, depends on the accelerating voltage, spot size, scanning speed and vacuum level. SEM has been used to evaluate the number density of canaliculi in human bone (Marotti et al., 1995). Resin-casted and acid-etched samples have been examined using SEM, showing the distribution, size and surface details of osteocyte lacunae and the vasculature (Fig. 9) (Feng et al., 2006; Kawakami et al., 2009; Kubek et al., 2010). Although SEM images have a characteristic depth of field and convey a 3D impression, owing to the fact that SEM can produce images in focus over a range of distances, the technique is essentially 2D. 
Fig. 8. Transmission electron microscopy tomography (TEM tomography). A 3D reconstruction of both the $\mathrm{ON}$ and the LCN based on TEM tomography data. Images (ac) show an osteocyte process in orange within a translucent canalicular wall in blue. Images (d-f) show the same views with collagen fibrils in green and red and a solid canalicular wall. Images (g-i) show the same views with a translucent canalicular wall. From (Kamioka et al, 2012) with kind permission from The Royal Society of Chemistry.

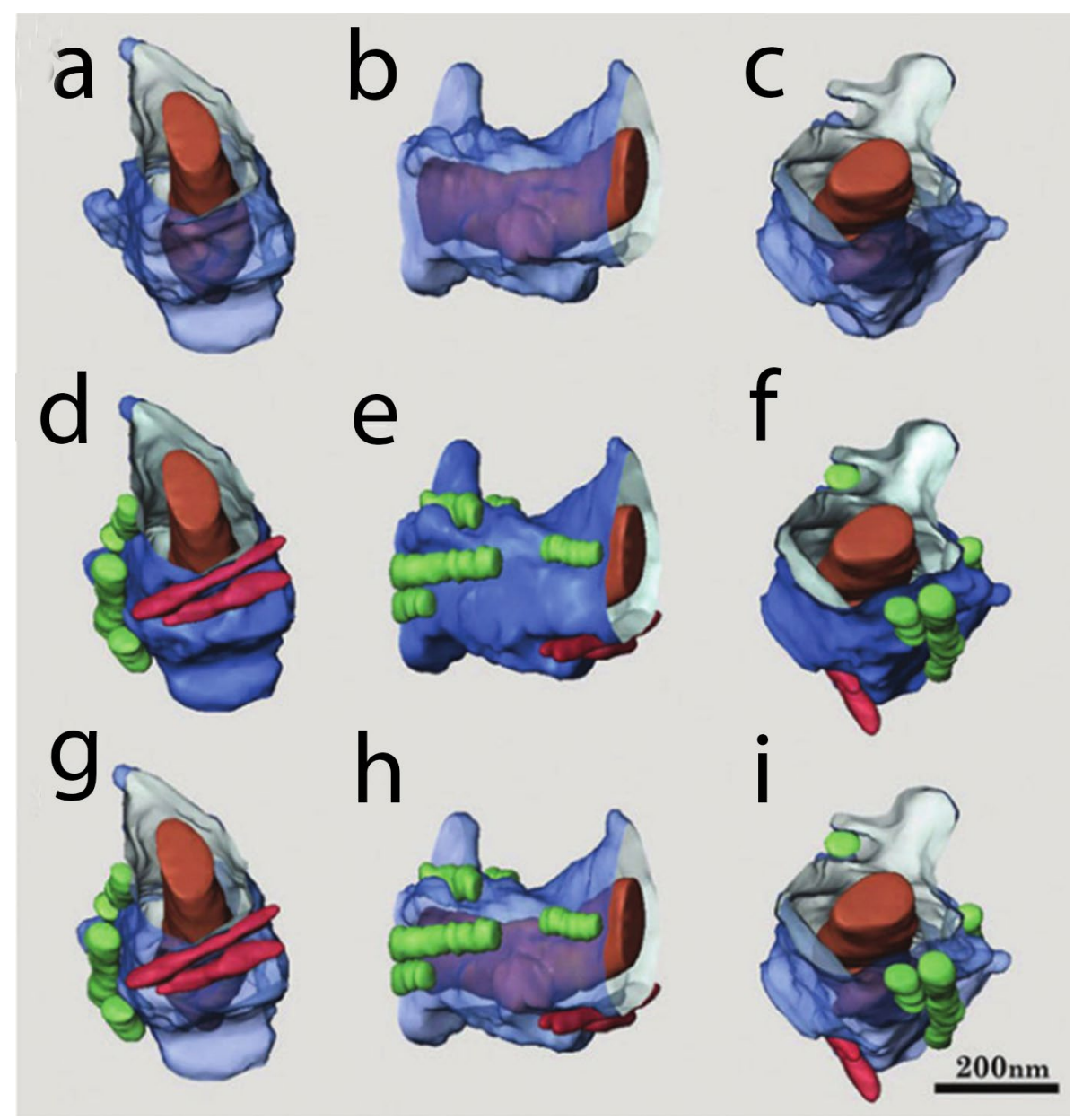

Fig. 9. Scanning electron microscopy (SEM). SEM image of an acid-etched plastic embedded section of mouse femur showing osteocyte lacunae and canalicular processes. From (Kubek et al., 2010) with kind permission from Wiley (online publishing).

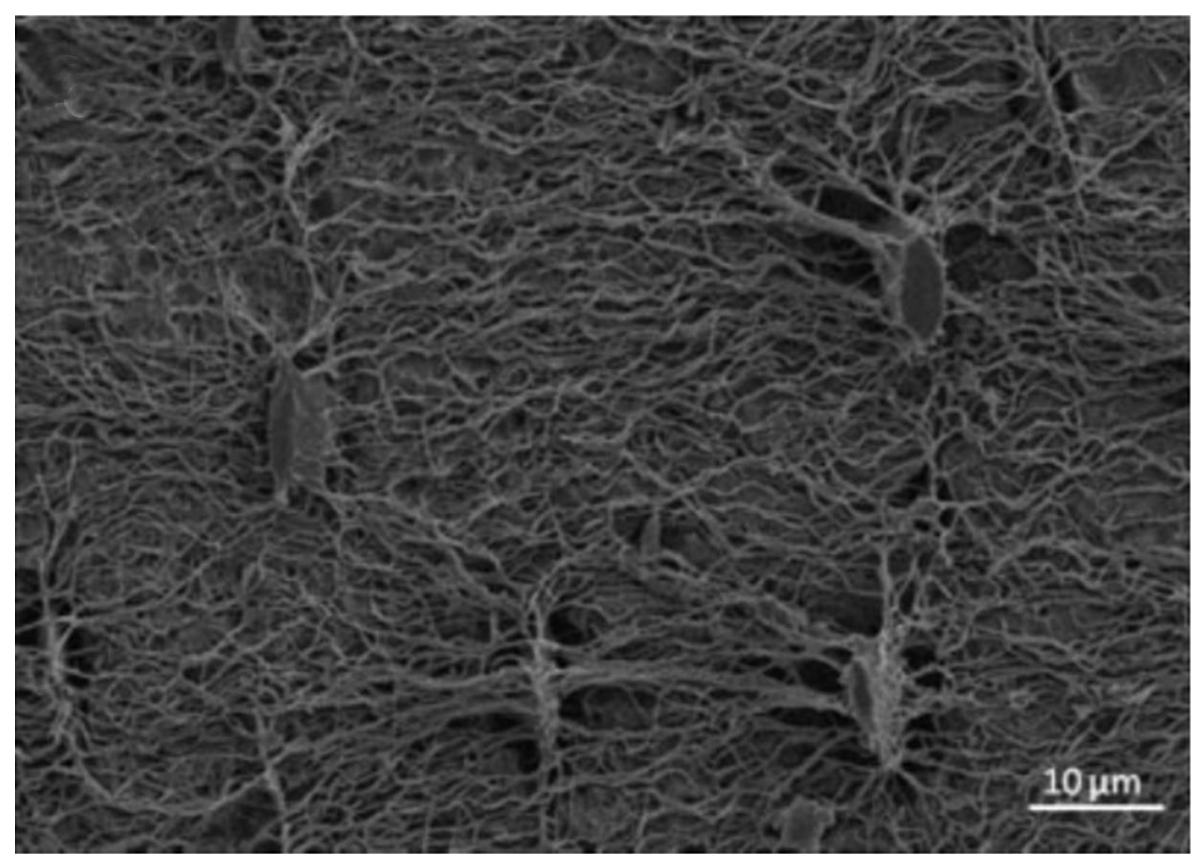

\section{Volume SEM}

Serial section SEM or 'array tomography' is a complex technique where multiple ribbons of serial ultrathin sections of tissue are collected on a substrate and subsequently viewed by SEM (Wacker and Schroeder, 2013). This technique is suitable for examining large areas at high $x$ - $y$-resolution ( $z$-resolution is limited by the section thickness). The sections can be re-examined many times (Tapia et al., 2012). Currently in development as a commercial product, the automated tape-collecting ultramicrotome (ATUM) facilitates the collection of serial sections on conductive tape for subsequent SEM imaging (Wacker et al., 2015).

Both serial FIB SEM and SBF SEM produce highresolution $3 \mathrm{D}$ data using automated sectioning techniques. $\mathrm{x} / \mathrm{y}$-resolution is comparable to TEM $(<5 \mathrm{~nm})$, whereas the 
Fig. 10. Serial focused ion beam scanning electron microscopy (serial FIB SEM). (a) A 3D representation of the LCN of the mid-diaphysis of a murine femur produced by serial FIB SEM. Osteocyte lacunae are shown as yellow ellipsoids and canaliculi in green. $(\mathbf{b}, \mathbf{c})$. The junction of an osteocyte lacuna and a canaliculus with the canalicular bone interface is shown in red. From (Schneider et al., 2011) with kind permission from Elsevier.

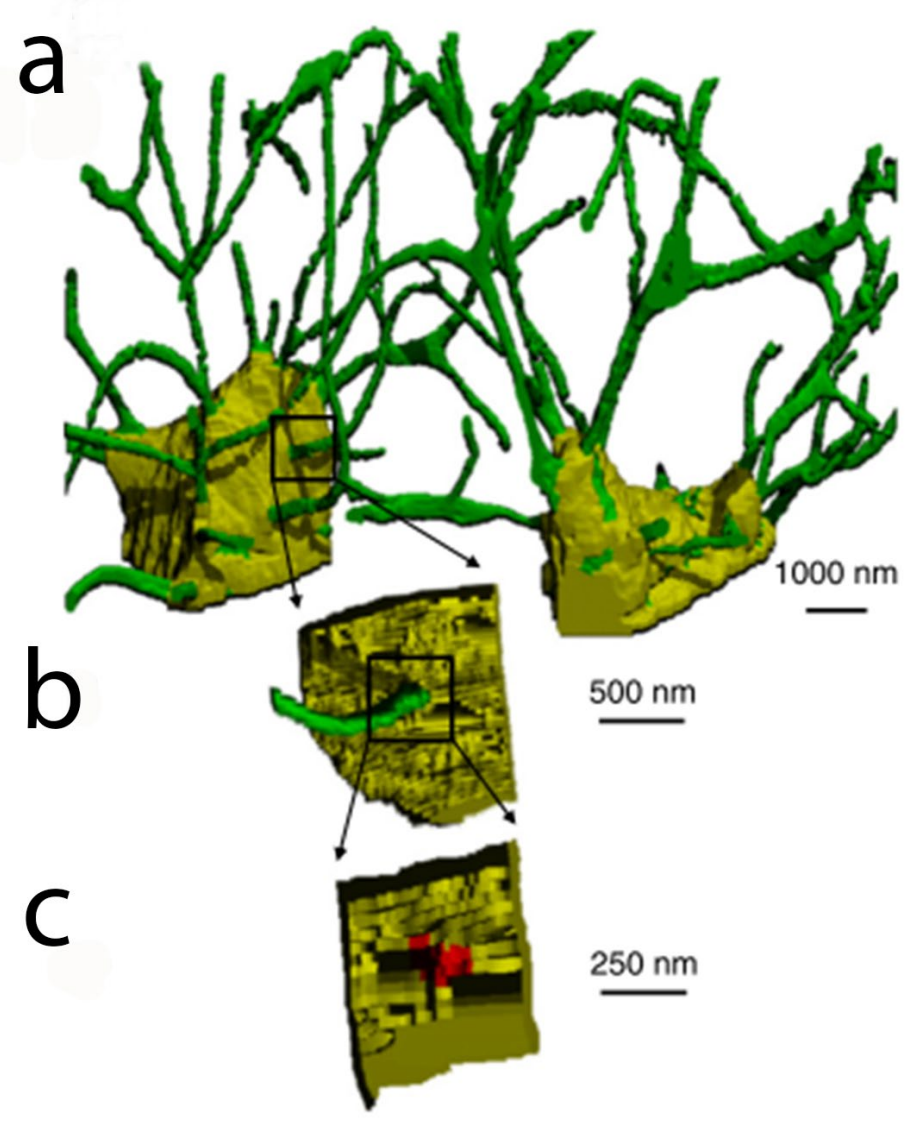

z-resolution is limited by the sectioning technique, typically $10 \mathrm{~nm}$ for serial FIB SEM and $15 \mathrm{~nm}$ for SBF SEM. In serial FIB SEM a focused ion source (usually gallium) is used to mill the resin-embedded tissue by scanning over the surface. The block-face is imaged using SEM and the process repeated until the volume of interest has (partly) been milled away. A proof-of-concept study by Schneider et al. for serial FIB SEM on bone tissue has successfully imaged parts of two adjacent osteocyte lacunae (Fig. 10) (Schneider et al., 2011). SBF SEM studies of bone tissue have not been reported, but it offers a promising $3 \mathrm{D}$ imaging approach since initial work in the neuroscience field a decade ago (Denk and Horstmann, 2004). Using a remotely controlled ultramicrotome within an SEM, nanoscopic layers are removed from the tissue block, which is consecutively assessed by a back-scattered electron detector to extract morphologies from 3D SEM stacks with spatial resolutions comparable to TEM. SBF SEM can cover large fields of view $\left(\sim 1 \mathrm{~mm}^{2}\right)$ and in principle, provides imaging contrast for soft and hard tissues at the same time, such as the ON\&LCN, respectively.

\section{X-ray imaging}

Conventional X-ray computed tomography (CT)

Unlike light, X-rays penetrate bone easily and without causing image artefacts. Attenuation of X-rays by bone tissue provides image contrast for (2D) X-ray projections at different angular positions, which are used to reconstruct the 3D map of the specimen. Sample preparation requirements are minimal, the technique is non-destructive and once set up, operator involvement is low. Bone can be imaged and analysed at different levels (organ, tissue, cellular) using micro-computed $\mathrm{CT}(\mu \mathrm{CT})$ at isotropic resolutions down to $1 \mu \mathrm{m}$ and below (Müller, 2009). It is notable that CT studies do generally not provide soft tissue contrast; reconstructions depict the $\mathrm{LCN}$ only, which is the negative imprint of the mineralised bone tissue surrounding the $\mathrm{ON}$.

Lab-based X-ray $\boldsymbol{\mu C T}$. Lab-based X-ray $\mu \mathrm{CT}$ systems are commonly used to investigate bone microstructure (Müller, 2009; Draenert et al., 2012; Peyrin et al., 2014). A range of spatial resolutions from about $100 \mu \mathrm{m}$ to below $1 \mu \mathrm{m}$ can be reached, comparable to the resolution obtained using LM, but not reaching the resolution of EM. Thus, $\mu \mathrm{CT}$ allows visualisation of lacunae, but not the fine detail of canaliculi. Commercially available $\mu \mathrm{CT}$ systems with improved spatial resolution have recently become available, some of which claim voxel sizes in the order of $100 \mathrm{~nm}$ at volumes of $1 \mathrm{~mm}$. Studies using this equipment have investigated differences in morphology of lacunae from different sites in the mouse (Vatsa et al., 2008) and compared morphology of lacunae in humans with different bone diseases, where significant variations have been observed between osteoarthritis, osteopenia and osteopetrosis (Vatsa et al., 2008; van Hove et al., 2009) (see also Table 1). A disadvantage of CT at these particular low voxel sizes below the micrometre range is the long acquisition time of many hours that are required for a signal-to-noise ratio that is sufficient to resolve individual osteocyte lacunae. 

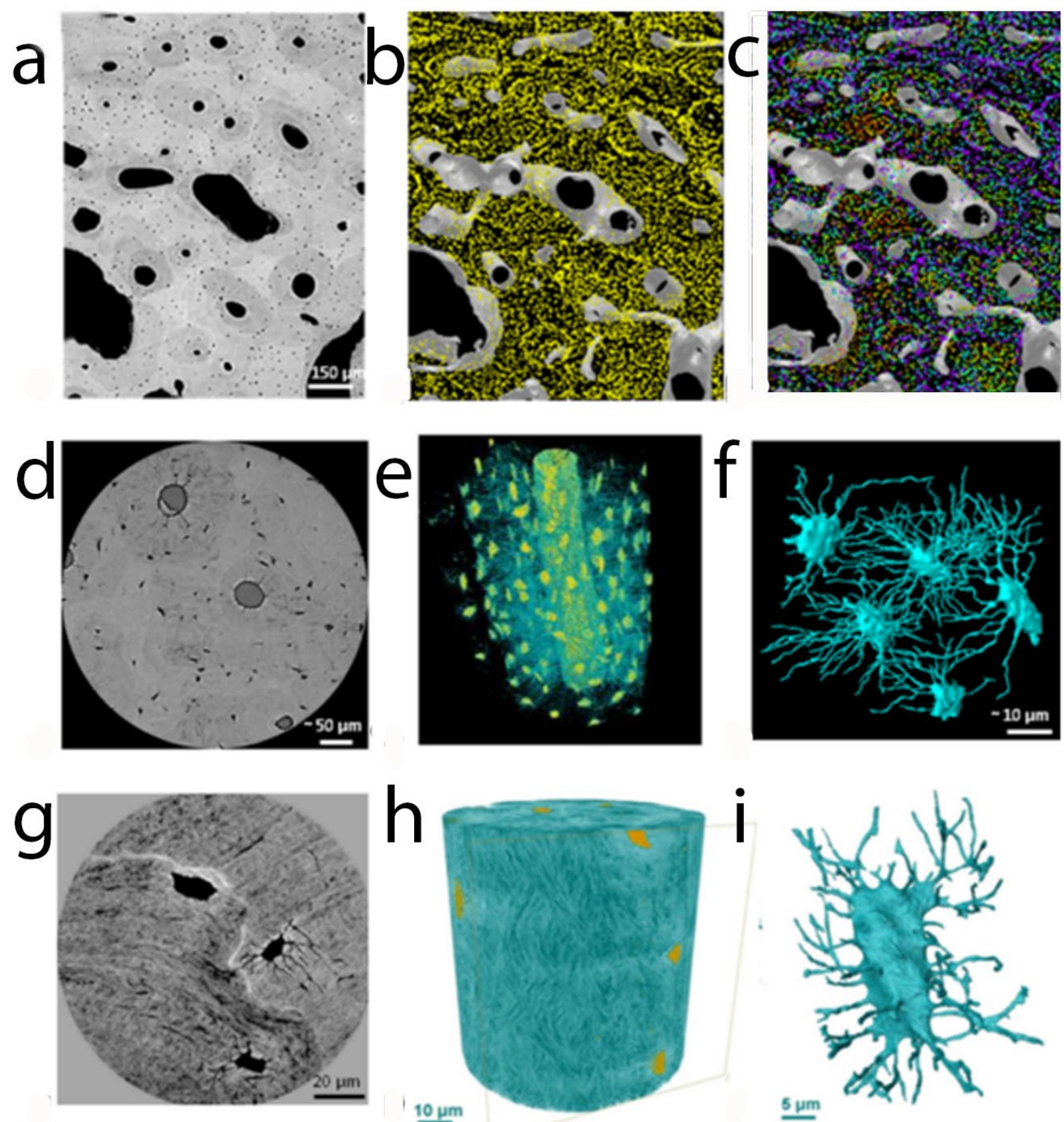

Fig. 11. Synchrotron radiation-based computed tomography (SR CT). SR CT images of human femur acquired at three different spatial resolutions. (a) Shows a CT slice showing osteocyte lacunae as small black dots, (b) illustrates the osteocyte lacunae (yellow) after 3D rendering and (c) shows a coloured map of the lacunae indicating their aspect ratio. (d) Displays a region of interest in the original CT slice at smaller voxel size, showing osteocyte lacunae and canaliculi, (e) a 3D rendering of the LCN (lacunae = yellow, canaliculi = cyan) and (f) a segmented 3D data set of five lacunae and their canaliculi. (g) Shows a high-resolution phase CT section with lacunae and canaliculi in black, (h) a $3 \mathrm{D}$ rendering, revealing an apparent texture of the collagen fibres (lacunae in orange) and (i) detailed rendering of one lacuna and its canaliculi. From (Peyrin et al., 2014) with kind permission of Springer Science and Business Media.

Synchrotron radiation-based CT (SR CT). Synchrotron sources, operated as large-scale electron accelerators at a small number of sites worldwide (http://www.lightsources.org/regions), offer quasimonochromatic X-rays and much higher photon fluxes with small X-ray source spot sizes, thereby providing spatial resolutions sufficient to resolve trabecular and internal bone microstructures on a sub- $\mu \mathrm{m}$ scale (Salome et al., 1999).
SR CT has been used to investigate trabecular architecture and osteocyte lacunae (Fig. 11) (Peyrin et al., 1998; Schneider et al., 2007). In addition to X-ray absorptionbased CT, coherent synchrotron light also provides phase contrast imaging capability, where the induced phase shift by the sample is exploited in comparison to the use of X-ray absorption only, generally involving a phase retrieval step before actual CT reconstruction. Phase contrast-based CT 
Fig. 12. Transmission X-ray microscopy CT (TXM CT). (a) 3D tomographic reconstruction of TXMCT data of a lacuna and surrounding canaliculi from mouse tibia trabecula. Scale bar $=5 \mu \mathrm{m}$. (b,c) Reconstructed slices showing inner structure and larger processes extending from the lacuna. Scale bars $=2 \mu \mathrm{m}$. From (Andrews et al., 2010) with kind permission of Cambridge Journals.

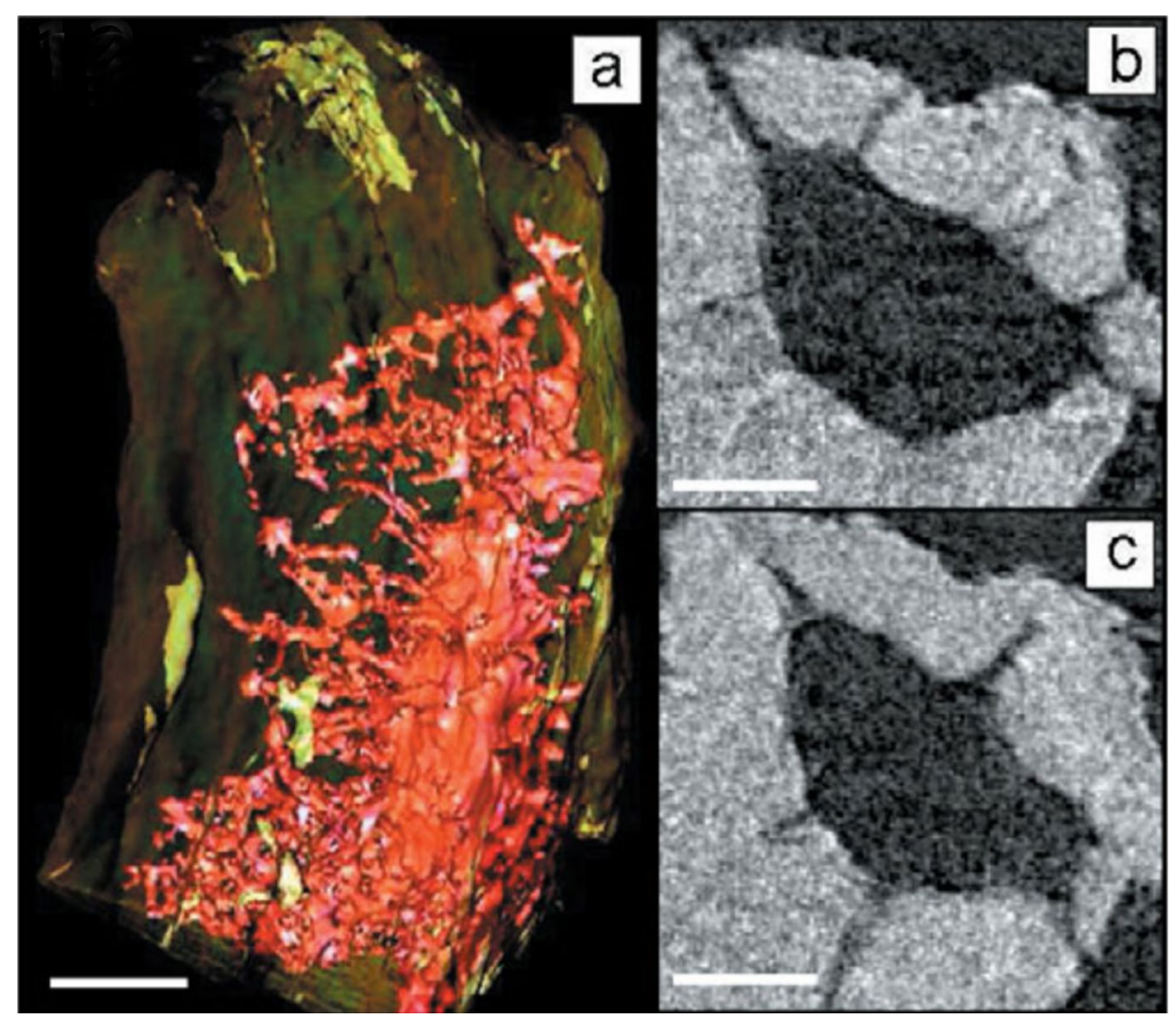

Fig. 13. X-ray ptychographic CT. Osteocyte lacuna retrieved from ptychographic CT imaging of a mouse femur (mid-diaphysis). (a) Volume rendering with the bone matrix in translucent colours to show the osteocyte lacunae (L) and connecting canaliculi (C). (b) Isosurface rendering of the LCN. Long edges of $3 \mathrm{D}$ scale bars $=5 \mu \mathrm{m}$. From (Dierolf et al., 2010) with kind permission of Nature Publishing Group.

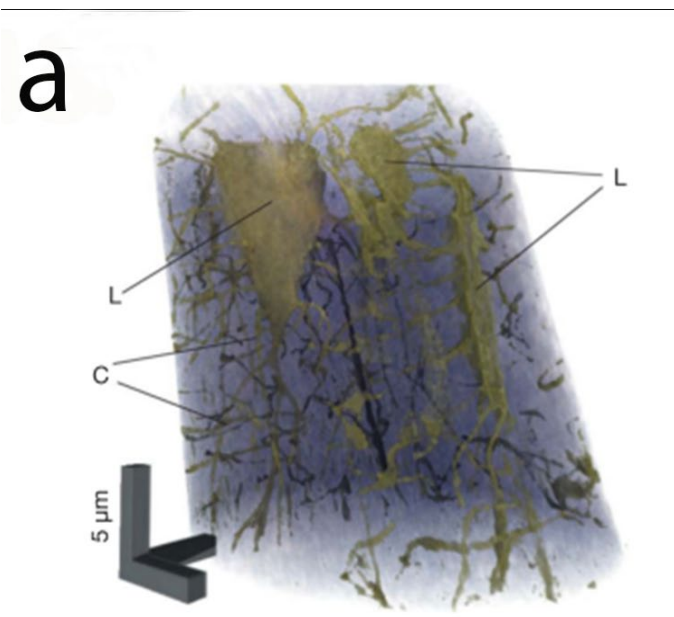

b

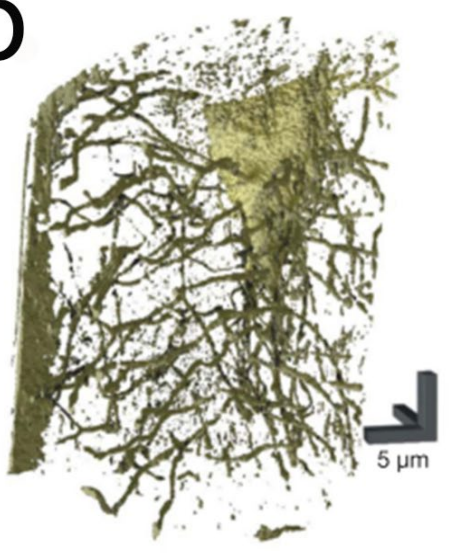

imaging yields images with improved contrast, particularly useful for weakly X-ray absorbing portions of the studied object such as collagen. Experimental SR-based imaging techniques include transmission X-ray microscopy (TXM), ptychographic X-ray CT and X-ray phase tomography (holotomography)/X-ray phase nanotomography.

Transmission X-ray microscopy (TXM). In TXM a zone plate acts as an X-ray lens to magnify the image onto the detector, which overcomes the limited spatial resolution of conventional $\mu \mathrm{CT}$, given by the diffraction limit of visible light around $200 \mathrm{~nm}$. TXM has produced $3 \mathrm{D}$ reconstructions of single osteocyte lacunae and associated canaliculi at reported spatial resolutions below $50 \mathrm{~nm}$, (Fig. 12), along with information regarding the composition of the surrounding bone matrix, for small fields of view (15-30 $\mu^{2}$ ) (Andrews et al., 2010). However, the canaliculi were interrupted, indicating that the provided spatial resolution of TXM needs improvement for a reliable assessment of the LCN.

Ptychographic X-ray CT. Ptychographic X-ray CT is a lensless imaging technique, which involves recording a sequence of X-ray diffraction patterns from a sample, exploiting the high phase sensitivity to produce quantitative and absolute 3D electron density maps (Dierolf et al., 2010). Excellent spatial resolution, presently $16 \mathrm{~nm}$ (Holler et al., 2014), at high contrast levels is possible, while the volume of interest is restricted to about $100 \mu \mathrm{m}^{3}$ and scanning times can be very long (hours up to days in extreme cases). In a study using X-ray ptychographic CT the LCN has been imaged at a voxel size of $65 \mathrm{~nm}$, showing continuous canaliculi (Fig. 13) (Dierolf et al., 2010). 
Fig. 14. X-ray phase tomography (holotomography)/X-ray phase nanotomography. (a) 3D rendering of the LCN of human femur (middiaphysis) assessed by X-ray phase tomography (holotomography)/ $\mathrm{X}$-ray phase nanotomography overlaid on a single greyscale slice. (b) Higher magnification of a single lacuna from (a) showing the branching of canaliculi. From (Langer et al., 2012) with kind permission of PLoS One.
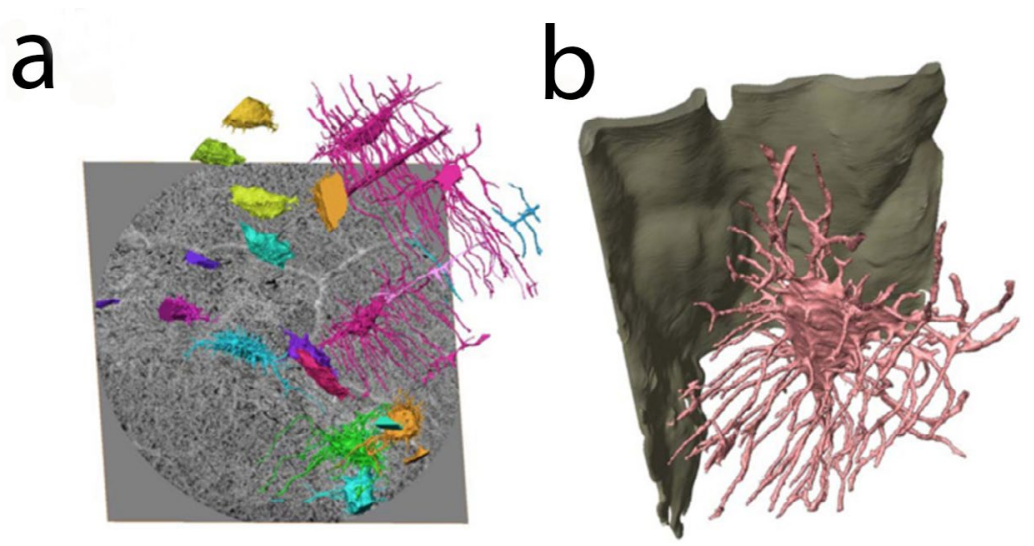

X-ray phase tomography (holotomography)/X-ray phase nanotomography. In X-ray phase tomography (holotomography)/X-ray phase nanotomography, developed as a SR imaging technique by a group operating at the European Synchrotron Radiation Facility (Langer et al., 2012), a focused monochromatic X-ray beam is used. The sample is placed behind the focal spot and imaged onto a scintillator and a camera situated at a fixed distance from the focal spot. Due to the divergence of the X-ray beam, moving the sample and recording the projections at varying distances from the focal spot varies the magnification factor. The $2 \mathrm{D}$ phase maps are retrieved following a holographic reconstruction approach (Cloetens et al., 1999), separating the object information from the defocused X-ray projections, which is employed for subsequent $\mathrm{CT}$ reconstruction to provide the $3 \mathrm{D}$ representation of the sample. X-ray phase nanotomography has been used by Langer and colleagues to image the $\mathrm{LCN}$ in 3D at a voxel size of $60 \mathrm{~nm}$ and a field of view of $90 \mu \mathrm{m}^{2}$ (Fig. 14) (Langer et al., 2012). Details of canalicular structure and branching, collagen fibre orientation and degrees of local bone mineralisation beyond microscopic scales were mapped in this study. X-ray phase nanotomography has also been applied by the same group to study the $3 \mathrm{D}$ orientation of mineralised collagen fibrils in human lamellar bone (Varga et al., 2013).

In summary, SR CT has been used to image and quantify the LCN, including image-based modelling approaches of bone mechanotransduction on sub-micrometre scales. Nonetheless, these newer X-ray CT imaging methods all operate for the moment at resolutions close to the dimensions of the relevant internal bone microstructures (e.g. osteocyte cell processes and canaliculi), and thus, care must be taken when interpreting X-ray CT image data with respect to bone mechanobiology.

\section{Scanning probe microscopy}

Atomic force microscopy (AFM) is a high-resolution scanning probe technique, which generates both images of surface topography and information on mechanical properties of tissues and materials. 2D images are recorded by scanning the surface of a sample (or a resin cast replica thereof) with a cantilever probe, where the cantilever deflection is measured with a laser. AFM can be used to image across a range of levels (tissue and cellular) and, where spatial resolutions of 20-50 $\mathrm{nm}$ can be reached in both air and liquid (Goldsbury et al., 2009; Usukura et al., 2012). AFM can resolve step heights of $>1 \mathrm{~nm}$ in crystal structures (Vavouraki et al., 2008). The ability to image in aqueous media is an important advantage of AFM, enabling closer to native-state imaging than other techniques. Additionally, it allows measurement of forces between molecules and investigation of mechanical properties (hardness, roughness) of biological materials. As the AFM does not have a lens system it does not suffer from the limitations in spatial resolution, given by the diffraction limit of visible light such as for LM or $\mu \mathrm{CT}$ for instance. Staining or coating of the sample is not required for AFM imaging. Compared to SEM, AFM offers a small field of view (in the order of $100 \mu \mathrm{m}^{2}$ ) and the scan times are longer. The occurrence of thermal drift makes measurement of distances less accurate. AFM studies have imaged the LCN and the collagen structure of the matrix (Fig. 15) (Reilly et al., 2001; Lin and $\mathrm{Xu}, 2011$; Katsamenis et al., 2013). In a recent study on rat femora AFM has been used to analyse the mineral phase of rat bone over the lifecourse for comparison with SEM, $\mu \mathrm{CT}$ and chemical analysis (Zhang et al., 2015). In addition, AFM has been used to investigate the crystal structure of the bone matrix (Tong et al., 2003; Kwon et al., 2009).

\section{Novel imaging methods}

Dual-purpose fluorescent and electron-dense labels for EM (SOG, miniSOG, APEX2) are genetically encoded tags for correlative light and electron microscopy (Shu et al., 2011; Lam et al., 2015), which could be applied to the ON\&LCN. Through-focus scanning optical microscopy (Attota et al., 2013), confocal soft X-ray scanning transmission microscopy (Spath et al., 2015) and intravital microscopy (Masedunskas et al., 2012; Alexander et al., 2013) - subcellular imaging in living animals - are techniques which may add to the repertoire for osteocyte investigation.

Our group has recently adopted SBF SEM to retrieve 3D data from the $\mathrm{ON}$ and the LCN simultaneously. We believe that this technique will facilitate the collection of quantitative 3D information by visualising the cell morphology and internal ultrastructure, the association with the PCM and ECM and the distribution of osteocytes within the bone matrix. Fig. 16 and Supplementary video 1 contain new data obtained from mouse bone, showing individual slices from the image stack and part of an osteocyte in a $3 \mathrm{D}$ reconstruction. 
Fig. 15. Atomic force microscopy (AFM). An AFM image of demineralised osteon in the midshaft of a bovine femur which reveals microstructural and ultrastructural features such as osteocyte lacunae, the canalicular network and lamellar interfaces. Adapted from (Katsamenis et al., 2013) with kind permission of Elsevier.

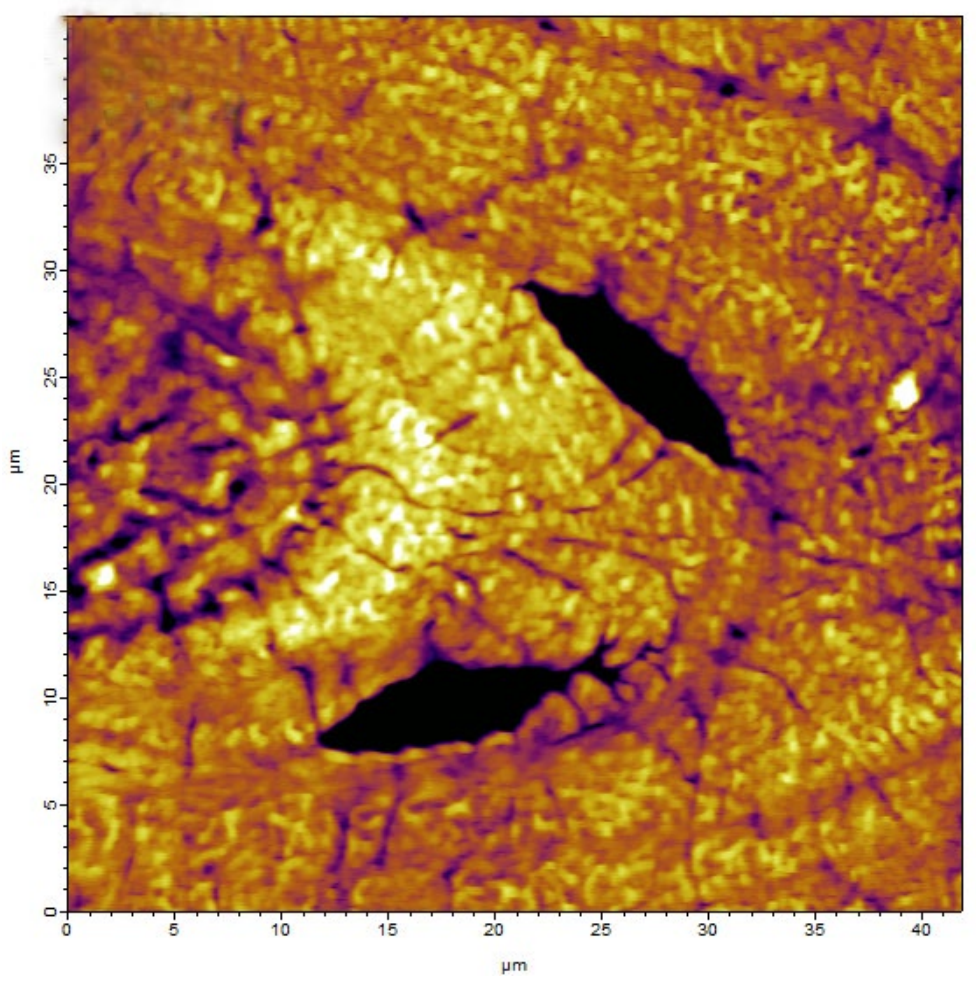

\section{Computational models}

A fundamental paradox exists in bone mechanobiology, where mechanical strains applied to the whole bone are much lower than the strain levels required to cause intracellular signalling. Therefore, whole-tissue strains must be amplified to elicit a biochemical response (You et al., 2000). In order to scrutinise this paradox, relevant mechanical pathways in bone mechanobiology must be understood.

\section{Proposed mechanisms for strain amplification}

Candidates for the mechanosensory system in bone were initially reviewed in the early 1990s, which included changes in whole tissue strain, hydrostatic pressure, and streaming potentials generated by fluid flow through pores in a charged matrix (Cowin et al., 1991). More recently, theoretical and experimental studies have provided more evidence in favour of interstitial fluid flow between the OC and the LCN, driven by extravascular pressure and loading and also of direct cell strain, where mechanical signals are transmitted from the whole-bone to the $\mathrm{ON}$ as opposed to hydrostatic pressure or streaming potentials. A further possibility is the sensation of fatigue microdamage which stimulates removal and/or repair of damaged bone.

\section{Interstitial fluid flow}

In terms of fluid flow, it was proposed in the 1970s that mechanical loading-induced fluid flow can facilitate cell nutrition and waste removal (Piekarski and Munro, 1977). Early mathematical models of stress-generated fluid flow in the LCN neglected the cell process and the PCM, while empty canaliculi were assumed (Kufahl and Saha, 1990). More refined models followed, which took into account both the PCM and the osteocyte processes. These models have shown that fluid flow is driven inwards to the centre of osteons and that a molecular sieve is present in the PCS (Zeng et al., 1994). This theoretical prediction was confirmed when tracer studies delineated the interstitial fluid space (Wang et al., 2004). Evidence for cellular excitation and mechanotransduction by the interstitial fluid flow model reviewed in (Fritton and Weinbaum, 2009) includes theoretical models, tracer experiments and computational fluid dynamics studies.

In the mid-1990s attention was focused on interstitial fluid flow as the key mechanism for bone mechanotransduction (Weinbaum et al., 1994; Cowin et al., 1995; Klein-Nulend et al., 1995). Investigators first suggested (Cowin and Weinbaum, 1998) and then developed (You et al., 2001) a model for strain amplification due to fluid flow for the mechanical stimulation of osteocyte processes. It was hypothesised that the cell process was like a cable attached to the bone matrix by proteoglycan tethering fibres and adhesion proteins. It was assumed that the glycosaminoglycans in the PCM are attached to the fibres. Further, it was proposed that during bone deformation, the fluid passing through the PCS creates a hydrodynamic drag on the bone matrix, which in turn creates tension on the tethering fibres and leads to strain that is experienced by the osteocyte process membrane. This model predicted cellular -level strain amplifications from tenfold to hundredfold. This finding corroborated earlier predictions (Weinbaum et al., 1994) with the difference that the activating signal was the fluid flow-induced drag on the tethering fibres, and not the fluid shear stress.

TEM studies subsequently revealed an organised actin filament bundle within the process and less organised actin within the cell body (Tanaka-Kamioka et al., 1998). 
Fig. 16. First serial blockface scanning electron microscopy (SBF SEM) images for bone tissue. Osteocyte from mouse tibia diaphysis imaged by SBF SEM. (a-c) Individual images of section numbers 0,40 and 80. (d,e) 3 D reconstruction of 150 SEM sections of an osteocyte from mouse bone visualised using Amira software (FEI, Hillsboro, Oregon, USA). Tissue prepared according to the 'Ellisman' protocol (Deerinck et al., 2010). Data assessed using the 3 View $^{\circledR}$ system of Gatan Inc. (Pleasanton, USA) within an FEI Quanta 250 FEG-SEM at $2.5 \mathrm{kV}$, vacuum $=40 \mathrm{~Pa}$, spot size $=3$, slice thickness $=50 \mathrm{~nm}$, pixel size $=22 \mathrm{~nm}$. Total volume imaged $=211 \mu \mathrm{m}^{3}$. Scale bars $=2 \mu \mathrm{m}$
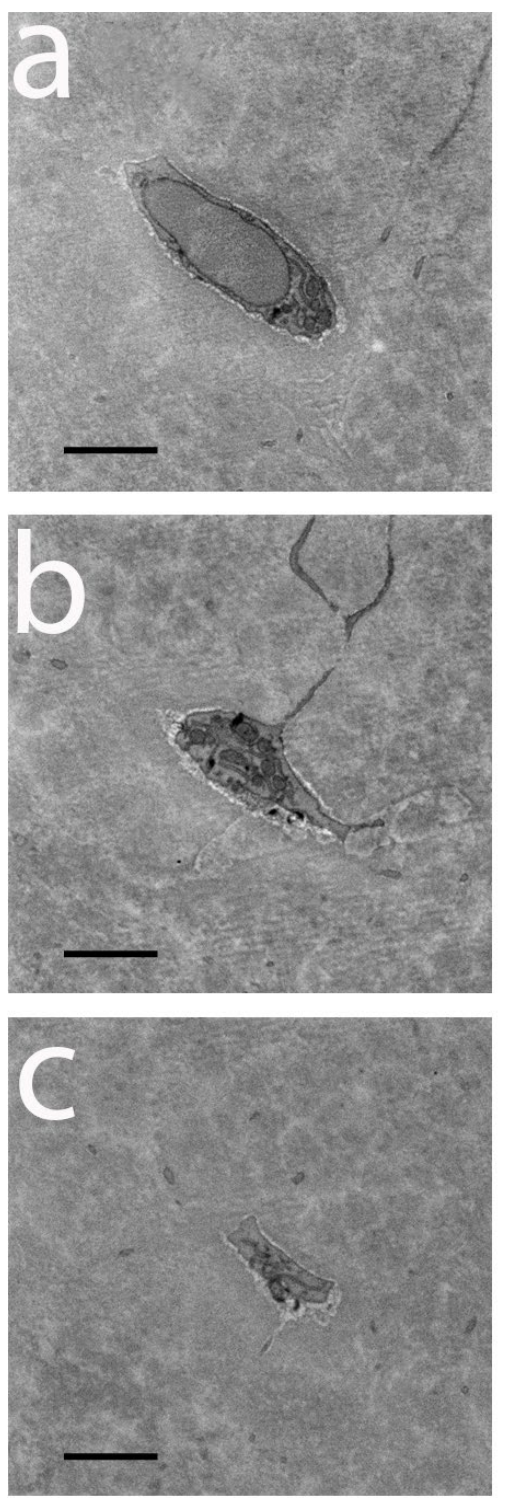

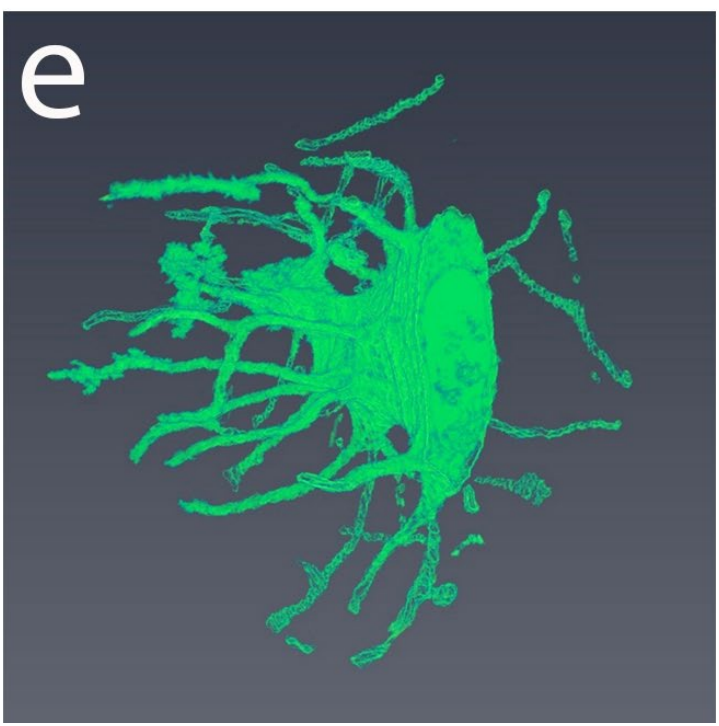

Modified sample fixation techniques using ruthenium resulted in improved images of both the central actin filament bundle and the PCM (You et al., 2004). Tethering elements were observed in these experiments, which had been hypothesised in the previous theoretical model (You et al., 2001). Evidence was also found that the PCM fills the whole space between the osteocyte and the bone matrix.

The original strain amplification model (You et al., 2001) assumed a radial arrangement of actin filaments, where the structural details of the cross-linking of the filaments were simplified. The improved ultrastructural mapping (You et al., 2004) led to a structurally more refined model (Han et al., 2004). Drawing on this refined model for strain amplification, the fundamental paradox in bone mechanobiology mentioned earlier (You et al., 2000) could directly be addressed, by demonstrating that mechanical strains applied to the whole bone can be amplified enough to elicit cell signalling and namely, that the stiffness of the actin filament bundle is three times higher than previously predicted (You et al., 2001).

None of the molecules mentioned in the studies of the original and the refined strain amplification model (You et al., 2001; Han et al., 2004) (CD44, proteoglycans, hyaluronic acid) are known to initiate intracellular signalling. This led to an investigation into integrinbased focal adhesion complexes on the cell processes, as they have been identified as mechanical transducer sites in other cells. A TEM study of mouse bone identified conical structures protruding from the ECM, which were connected with the membrane of the osteocyte process (Wang et al., 2007) (see Fig. 3). Immunohistochemical analysis revealed punctate staining of $\alpha \mathrm{V} \beta 3$ integrins on the cell process and $\beta 1$ integrins on the cell body but absent from the processes. Additionally, tethering fibres were co-located with the ECM projections (McNamara et al., 2009). The creation of a new strain magnification model was thus triggered, introducing the concept of integrin-based initiation of intracellular signalling (Wang et al., 2007) (see Fig. 3). The model exhibits rigid integrin attachments located at the tip of the conical structures, the tethering complex at the osteocyte process membrane surface linked to the central actin bundle by cross-linking molecules, and actin filaments that slide relative to each other. The motion of the actin and the flexibility of the 
tethering elements allow local stress concentrations and large axial strains to build up in the vicinity of the more rigid integrin attachment sites when there is interstitial fluid flow through the PCM. This model leads to greatly amplified axial strains in the membrane near the integrin attachments, which are sufficient to open stretch-activated ion channels and to initiate electrical signalling. The role of $\alpha \mathrm{V} \beta 3$ integrin attachments has been further investigated using cultured cells showing that blocking integrin function changes osteocyte morphology and disrupts the release of biochemical responses to shear stress (Haugh et al., 2015). A finite element (FE) model using focal adhesion density and stiffness measurements from AFM showed that the density of focal adhesions is related to the cell morphology and that both focal adhesion density and morphology can modulate the effect of substrate stiffness on internal cell tension (Mullen et al., 2014). It has been suggested by analytical models that the viscosity of the interstitial fluid and the permeability of the PCM significantly affect flow in the LCN (Sansalone et al., 2013). Models based on UHVEM predict that the geometry of the PCS affects the velocity of fluid flow (Kamioka et al., 2012). Fluidstructure interaction (FSI) studies take into account the elasticity and potential deformation of the cell membrane in response to fluid flow. CLSM data has been used with FSI modelling to predict the mechanical environment of osteocytes during loading and showed that stimulation is greater in the cell processes than the cell body (Verbruggen et al., 2014).

\section{Direct matrix-cell deformation}

While fluid flow theory is an important concept for bone mechanotransduction, an alternative mechanism is direct matrix-cell deformation (Nicolella et al., 2005). The effect of direct bone matrix deformation has been studied by in vivo strain measurements during physical activity (Hoshaw et al., 1997; Milgrom et al., 2004). The disadvantage of these measurements is that they do not account for microstructural inhomogeneity and variations in local tissue/cell strains (Nicolella et al., 2006). Therefore, FE models have been used to quantify local tissue deformation and response to changes in the local environment (Bonivtch et al., 2007; Deligianni and Apostolopoulos, 2008). It has been proposed that the osteocyte lacuna acts as a strain amplifier and that the amplification factor is related to the properties of the surrounding bone matrix (Nicolella et al., 2006; Bonivtch et al., 2007). Using FE modelling it was found that changes in the osteocyte (body and process) stiffness had little effect on strain levels but that changes in the material properties of the surrounding bone matrix influenced the level of strain transmitted to the osteocyte, with the maximum strains resulting from decreased tissue modulus (Bonivtch et al., 2007). In a recent publication (Wang et al., 2015b) a simplified 3D FE model of the ON\&LCN was used to explore the relationship between strain amplification and loading. The results from this study suggest that the strain amplification factor increases with loading frequency and increased strain.

A multilevel FE approach, using idealised LCN geometry, related loads applied to whole bone with strains at a single lacuna and canaliculi (Deligianni and
Apostolopoulos, 2008). This study revealed that osteocytes may undergo much larger strains than measured in vivo, which may be sufficient to elicit cell responses, suggesting that loads directly mediated through the mineralised bone matrix may produce a reaction similar to fluid flow.

\section{Microcrack theory}

Disruption or deformation of osteocytes by microcracks in the bone matrix caused by loading and fatigue has been shown to trigger firstly localised apoptosis and subsequently, targeted bone remodelling (Martin, 2000; Martin, 2002; Noble et al., 2003; Heino et al., 2009). A relationship between microdamage, osteocyte apoptosis and the initiation of bone resorption was demonstrated by showing that prevention of osteocyte apoptosis leads to complete inhibition of osteoclastic remodelling (Cardoso et al., 2009). Concomitantly, microcracks are likely to affect the fluid flow within bone and consequently, bone mechanotransduction and mechanosensation. In a study measuring microstructural strain in cortical bone based on light microscopy and digital image correlation, Nicolella and co-workers found that the presence of a microcrack caused strain amplification that is greatly increased (Nicolella et al., 2005). In a FE modelling study for a simplified Haversian system, where strain-induced interstitial fluid velocities in osteons have been evaluated, Nguyen and colleagues showed that the presence of a microcrack would reduce the fluid velocity, modifying the cellular environment and thus mechanobiological mechanisms (Nguyen et al., 2011). It is also suggested that microcracks do not cause a reduction in local signal sensation but a reduction in transduction (Ridha et al., 2015). Computational studies incorporating both mechanical strain and microdamage have been carried out. One such study has suggested that there is a damage threshold below which osteocyte processes can sense changes in strain and fluid flow, above which osteocyte signalling is disrupted and apoptosis occurs (McNamara and Prendergast, 2007). In line with that, a FE model based on ruptured osteocyte connections suggests that microcracks inhibit transmission of signals through the osteocyte network (Ridha et al., 2015).

\section{Proposed mechanotransduction mechanisms}

Mechanotransduction in the broader sense is the conversion of a mechanical signal into a biochemical response, which is transmitted to an effector cell, resulting in a coordinated response causing appropriate architectural changes. Osteocytes (processes and/or cell body) detect load via a mechanical signal, which is perceived by one or more of the mechanisms discussed above. Osteocytes respond to the mechanical signal with a range of biomolecules such as intracellular calcium signals, nitric oxide which modulates the activity of osteoblasts and osteoclasts, prostaglandins and Wnt proteins which stimulate osteoblast recruitment and activity, bone morphogenetic proteins which induce bone formation and sclerostin which inhibits bone formation reviewed in (Klein-Nulend et al., 2013). These signalling molecules modulate the recruitment and activity of osteoblasts and osteoclasts. The way how mechanical stimuli become processed and integrated by the osteocytes 
and their network, and transmitted as signals to osteoblasts and osteoclasts, is suspected to depend largely on the network topology and the precise network architecture and connectivity of the ON\&LCN. Moreover, in a novel perspective on the complexity of the ON\&LCN, Buenzli and Sims emphasise the extent of the mechanosensory system within bone and the large number of osteocytes in the human skeleton (several billions), comparable to the numbers of neurons in the brain (Buenzli and Sims, 2015). Most likely, this allows for a large capacity of information to be processed and transmitted by the ON, which should be explicitly addressed in the future.

\section{The importance of high-resolution 3D imaging to computational modelling in bone mechanobiology}

Many computational modelling studies on bone mechanotransduction are limited by the use of idealised ON\&LCN geometries, which have been shown to be inappropriate when investigating local strains (e.g. simplifying the lacuna as an ellipsoid) (McCreadie et al., 2004). Comparing the use of actual and idealised pericellular and canalicular geometries in computational fluid dynamics shows the influence of physiological geometry on force magnitudes imparted to cells through load-induced fluid flow. Idealisation resulted in underestimation of flow and shear stresses imparted by fluid drag on osteocytes (Anderson and Knothe Tate, 2008). A numerical study of murine osteocytes highlighted large differences between the results derived from idealised and from more realistic models for the ON\&LCN, the PCM and ECM (Verbruggen et al., 2012). However, LCN geometry in this study was approximated based on CLSM images, which offers a spatial resolution insufficient to capture the fine details of the canaliculi. In a FSI study the same authors applied CLSM on fluorescently stained rat bone sections to create a realistic ON\&LCN model, which was further informed by earlier TEM data in terms of the conical ECM projections (Verbruggen et al., 2014). This study predicted that the cell process is the most mechanically active and mechanosensitive region of the osteocyte (Verbruggen et al., 2014), confirming the results from an earlier study using an idealised model (Anderson et al., 2005). Some limitations of this study are the spatial resolution $(\sim 100 \mathrm{~nm})$ not being adequate to detect the narrower processes, and the use of 2D TEM data to create a 3D model. Furthermore, assumptions were made about material properties of the cell and the bone matrix, the PCM was excluded and the anisotropy of the cell and the influence of the actin cytoskeleton were not reflected in the model. This does not undermine the value of the study of Verbruggen and colleagues, but rather underlines the complexity of modelling mechanotransduction processes at cellular and sub-cellular scales.

As the reliability of FE analyses is largely determined by the accuracy of the model input, accurate spatial description and quantification of the ON\&LCN geometry is an essential prerequisite for more realistic models. In line with this, computational modelling of the LCN using high-resolution imaging techniques has been carried out. An LCN model for FE analysis has been derived from ptychographic X-ray CT data of mouse bone (Dierolf et al., 2010; Schneider et al., 2012), which provided evidence that high strain magnifications are limited to pericanalicular regions, and that FE modelling based on experimentally derived images of the LCN leads to higher strain magnification in comparison with idealised models.

A recent fluid dynamics modelling approach employed ultra-high voltage EM tomography to reconstruct a small section of a canaliculus in $3 \mathrm{D}$ (ignoring tethering elements and proteoglycans in the PCM) (Kamioka et al., 2012). Contrary to previous work (Wang et al., 2007; McNamara et al., 2009), no conical projections from the matrix wall were found (based on a single sample). In addition, the study of Kamioka and co-workers showed that variations in the roughness of the canalicular wall influenced the loading-induced flow of the interstitial fluid. One conclusion of this study was that fluid flow simulations based on 2D data may lead to inaccurate findings about mechanotransduction mechanisms.

In a recent FE study human bone was imaged using synchrotron X-ray phase nanotomography at a voxel size of $50 \mathrm{~nm}$ (Varga et al., 2014). The LCN morphology was quantified and the osteocyte and processes artificially reconstructed using a priori assumptions from TEM and CLSM studies to approximate cell shape, canalicular occupation as well as PCM and osteocyte process dimensions. FE models were generated, which were subjected to uniaxial compression, confirming the existence of strain magnification through direct matrix-cell mechanotransduction. Furthermore, it was reported in the same study that cell deformations were localised at the cell-process junctions. These results confirm findings of previous investigations (Bonivtch et al., 2007; Deligianni and Apostolopoulos, 2008). A limitation of this study was the lack of original imaging data describing the cell shape.

In conclusion, reliable quantitative characterisation of both the $\mathrm{ON}$ and the LCN based on advanced 3D imaging across different length scales will inform computational models of bone mechanotransduction and will allow realistic predictions of this complex dynamic system and its emergent behaviours (Buenzli and Sims, 2015). For example, data on canalicular occupancy and the number of cells, which make connections with vascular and other structures, would improve accuracy when calculating the number of connections between osteocytes and related cell networks.

\section{The future role of high-resolution 3D imaging in osteocyte anatomy and pathology}

Much research has been carried out into microcracks, loadinduced fluid flow and direct strain from the matrix to the osteocyte and their posited roles in mechanotransduction and mechanosensation ; however, many issues still remain to be discovered and thoroughly investigated. It is necessary to clarify the role of (ultra)structural differences between the cell processes and the cell body of osteocytes in sensation of mechanical stimuli and in the initiation of cell signalling. The involvement of primary cilia is unclear and it is not known whether it is crucial for bone mechanosensation. The co-localisation of integrins with 
Table 3. Choice of imaging technique for the assessment of the osteocyte and lacuno-canalicular network. Abbreviations: Transmission electron microscopy (TEM), micro-computed tomography $(\mu \mathrm{CT})$, confocal laser scanning microscopy (CLSM), serial focused ion beam scanning electron microscopy (serial FIB SEM), serial block face scanning electron microscopy (SBF SEM).

\begin{tabular}{|l|c|c|c|c|c|}
\hline Choice of imaging technique & 3D & High resolution & Automated & Hard and soft tissue contrast & Volume of interest \\
\hline Serial section TEM & yes & yes & no & yes & $>10^{3} \mu \mathrm{m}^{3}$ \\
\hline $\boldsymbol{\mu C T}$ & yes & intermediate & yes & no & $>10^{9} \mu \mathrm{m}^{3}$ \\
\hline CLSM & yes & no & no & yes & $0.1 \mathrm{~mm}^{3}$ \\
\hline X-ray ptychographic CT & yes & intermediate & yes & no & $>10^{3} \mu \mathrm{m}^{3}$ \\
\hline Serial FIB SEM & yes & yes & yes & yes & $10^{4} \mu \mathrm{m}^{3}$ \\
\hline SBF SEM & yes & yes & yes & yes & $10^{5} \mu \mathrm{m}^{3}$ \\
\hline
\end{tabular}

the protrusions, connecting the cell membrane and the mineralised bone matrix, also requires further investigation.

Data from recent research, where new imaging techniques have been employed, were used by Buenzli and Sims (Buenzli and Sims, 2015) for theoretical mathematical calculations of the dimensions of the entire $\mathrm{ON}$ and LCN in an average human adult skeleton. This has led to insights into the extent of the influence of osteocytic osteolysis on bone and its potential to alter the structural properties and permeability of the ON\&LCN. The use of cutting-edge techniques, such as UHVEM, has raised questions about the impression produced by $2 \mathrm{D}$ imaging that osteocyte processes are directly attached to the canalicular walls (Kamioka et al., 2012) although the small field of view for UHVEM data has to be taken into account for such considerations.

Improvements will help to validate theoretical and computational models for strain magnification as well as quantifying bone interstitial fluid movement in response to mechanical loading. Questions to be addressed include: What is the 3D structure of the ON\&LCN at different sites of an entire bone sample? How are the ON and the LCN and thus, the interstitial fluid flow altered in disease? To answer these questions, existing 3D imaging techniques need refinement and further development, and novel highresolution 3D imaging techniques will be applied in the field of bone research.

\section{Refinements and developments of high-resolution 3D imaging techniques}

The features of a number of currently available 3D imaging techniques are set out in Table 3, showing the relative strengths of each as an imaging approach for the ON\&LCN. The number of potentially useful techniques is rising however, as imaging techniques traditionally applied in physics and materials science are increasingly applied to address biological and medical questions. Some recent developments include the improved resolution of CT techniques using synchrotron sources or the development of cryo-TEM (Weston et al., 2010; Ercius et al., 2015), which facilitates high-resolution imaging of samples in a near-to-native state. Cryo-soft X-ray tomography is a promising technique for close-to-native state imaging (Carzaniga et al., 2014; Wang et al., 2015a). Improvements in the sensitivity of EM detectors used in SBF SEM may reduce the amount of specimen preparation, needed and beam deceleration could allow imaging at lower energies, and therefore causing less specimen damage. Furthermore, conductive resins would improve the quality of SBF SEM data quality and it may even be possible to carry out SBF SEM on samples up to $10 \mathrm{~mm}$ in width (Marx, 2013; Ellisman, 2015). New refinements of super-resolution LM are breaking the diffraction limit of visible light (Betzig, 2015; Wang et al., 2015a). Other recent LM improvements include better depth penetration and reduction of light scattering (Wang et al., 2015a). The development of multibeam SEM with more than 60 parallel beams will greatly accelerate the rate of data collection (Marx, 2013). A multienergy deconvolution SEM is currently in development which can perform 'sub-surface' imaging using different energy beams, which improves $z$-resolution and reduces the risk of missing fine structures (Marx, 2013).

Techniques previously confined to the realm of engineering are increasingly being applied to biological systems and pathology research (Friedl et al., 2012). For example, electrochemical strain microscopy (a variation of AFM) captures dynamics in nanometre scale volumes (Jesse et al., 2012) and ferroelectric switching has been observed in ECM components (Liu et al., 2014).

\section{Conclusions and outlook}

At present, high-resolution 3D native-state imaging of the ON\&LCN is impossible. Achieving the goal of concurrent imaging of both hard and soft tissue networks would undoubtedly have a significant impact on the understanding of bone adaptation and pathology processes. Many new high-resolution 3D imaging techniques are advancing the ability to achieve this aim incrementally.

Given that mechanotransduction mechanisms act across scales (from the whole-bone down to the cell ultrastructure) bone tissue must be assessed at different hierarchical levels (Lucas et al., 2012). No technique exists that can cover so many different scales at the same time, due to physical limitations. Even to image the different features of the ON\&LCN in a restricted field of view, several length scales must be covered, from the osteocyte and the lacuna $(\sim 10 \mu \mathrm{m})$ down to the cell processes and canaliculi $(<1 \mu \mathrm{m})$, the PCS $(<100 \mathrm{~nm})$, etc. A correlative imaging approach combining light, X-ray and electron microscopy modalities might prove to be useful. Large volumes could be analysed and high-resolution quantitative information obtained and used for realistic in silico simulations. 
Comparison of 3D quantitative hallmarks of osteocytes in healthy and pathological tissue would enhance the current knowledge of mechanotransduction processes. The wealth of current data on bone physiology and the $\mathrm{ON}$ and LCN indicate the importance of a multi- and inter-disciplinary approach, combining the expertise and differing experimental methodologies of life scientists, microscopists, engineers and mathematicians.

We have identified relevant phenotypes of developing, ageing, healthy and diseased bone on cellular, sub-cellular and ultrastructural levels. Techniques used for ON\&LCN imaging have been discussed and assessed, including their relevance for computational modelling approaches. In conclusion, we have considered how developments in high-resolution 3D imaging technology and novel imaging approaches may contribute to the study of osteocyte structure. First attempts to visualise the ON\&LCN using SBF SEM have been reported. As high-resolution 3D imaging techniques mature, become more widely available and are used to image bone from different species, ages, and (pathological) states, the nature of the elusive role of the osteocyte for bone development, ageing, health and disease will become clearer in the future.

\section{Acknowledgements}

This work is funded by the Institute for Life Sciences and the Faculty of Engineering and the Environment at the University of Southampton. Funding to ROCO from the Medical Research Council, the Engineering and Physical Sciences Research Council and the Biotechnology and Biological Sciences Research Council UK Regenerative Medicine Platform Hub Acellular Approaches for Therapeutic Delivery (MR/K026682/1) and BBSRC LO21071/ and BB/L00609X/1 is gratefully acknowledged. The authors thank Mr. Matthew Sharp, Clinical Neurosciences, University of Southampton, for supplying the murine tissue under Home Office Project Licence Number 30/3095. Dr. Orestis Katsamenis supplied the image for Fig. 15. Dr. David Johnston provided advice and fruitful conversations on confocal laser scanning microscopy imaging. We would also like to specially thank the reviewers for their exceptional and comprehensive analysis and insightful comments, which have greatly improved the manuscript.

\section{References}

Adachi T, Aonuma Y, Tanaka M, Hojo M, TakanoYamamoto T, Kamioka H (2009) Calcium response in single osteocytes to locally applied mechanical stimulus: differences in cell process and cell body. J Biomech 42: 1989-1995.

Alexander S, Weigelin B, Winkler F, Friedl P (2013) Preclinical intravital microscopy of the tumour-stroma interface: invasion, metastasis, and therapy response. Curr Opin Cell Biol 25: 659-671.

Anderson EJ, Kaliyamoorthy S, Iwan J, Alexander JI D, Knothe Tate ML (2005) Nano-microscale models of periosteocytic flow show differences in stresses imparted to cell body and processes. Ann Biomed Eng 33: 52-62.

Anderson EJ, Knothe Tate ML (2008) Idealization of pericellular fluid space geometry and dimension results in a profound underprediction of nano-microscale stresses imparted by fluid drag on osteocytes. J Biomech 41: 17361746.

Andrews JC, Almeida E, van der Meulen $\mathrm{MCH}$, Alwood JS, Lee C, Liu Y, Chen J, Meirer F, Feser M, Gelb J, Rudati J, TkachukA, Yun W, Pianetta P (2010) Nanoscale $\mathrm{X}$-ray microscopic imaging of mammalian mineralized tissue. Microsc Microanal 16: 327-336.

Atkins GJ, Findlay DM (2012) Osteocyte regulation of bone mineral: a little give and take. Osteoporos Int 23: 2067-2079.

Attota R, Bunday B, Vartanian V (2013) Critical dimension metrology by through-focus scanning optical microscopy beyond the $22 \mathrm{~nm}$ node. Appl Phys Lett 102: 222107.

Balemans W, Ebeling M, Patel N, Van Hul E, Olson P, Dioszegi M, Lacza C, Wuyts W, Van Den Ende J, Willems P, Paes-Alves AF, Hill S, Bueno M, Ramos FJ, Tacconi P, Dikkers FG, Stratakis C, Lindpaintner K, Vickery B, Foernzler D, Van Hul W (2001) Increased bone density in sclerosteosis is due to the deficiency of a novel secreted protein (SOST). Hum Mol Genet 10: 537-543.

Barbour EP (1950) A study of the structure of fresh and fossil human bone by means of the electron microscope. Am J Phys Anthropol 8: 315-329.

Becker CB (2014) Sclerostin inhibition for osteoporosis - a new approach. N Engl J Med 370: 476-477.

Betzig E (2015) Single molecules, cells, and superresolution optics (Nobel lecture). Angew Chem Int Ed Engl 54: 8034-8053.

Billings B, Stern M, Bergman C, Register TC, Stern AR (2012) Aging Bone: Age effects on osteocyte lacunar and canalicular microarchitecture. In: Proceedings of AADR $41^{\text {st }}$ annual meeting of the American and Canadian Association for Dental Research, Tampa, Florida, 1459.

Bonetta L (2005) Zooming in on electron tomography. Nat Meth 2: 139-144.

Bonewald LF (2011) The amazing osteocyte. J Bone Miner Res 26: 229-238.

Bonewald LF (2013) Osteocyte biology. In: Osteoporosis (fourth edition) (Marcus R, Feldman D, Dempster DW, Luckey M, Cauley JA, eds), Academic Press, Elsevier, pp 209-234.

Bonewald LF, Johnson ML (2008) Osteocytes, mechanosensing and Wnt signaling. Bone 42: 606-615.

Bonivtch AR, Bonewald LF, Nicolella DP (2007a) Tissue strain amplification at the osteocyte lacuna: A microstructural finite element analysis. J Biomech 40: 2199-2206.

Brennan MA, Gleeson JP, O’Brien FJ, McNamara LM (2014a) Effects of ageing, prolonged estrogen deficiency and zoledronate on bone tissue mineral distribution. J Mech Behav Biomed Mater 29: 161-170.

Brennan MA, Haugh MG, O’Brien FJ, McNamara LM (2014b) Estrogen withdrawal from osteoblasts and osteocytes causes increased mineralization and apoptosis. Horm Metab Res 46: 537-545. 
Buenzli PR, Sims NA(2015) Quantifying the osteocyte network in the human skeleton. Bone 75: 144-150.

Burra S, Nicolella DP, Francis WL, Freitas CJ, Mueschke NJ, Poole K, Jiang JX (2010) Dendritic processes of osteocytes are mechanotransducers that induce the opening of hemichannels. Proc Natl Acad Sci USA 107: 13648-13653.

Bushong EA, Johnson DD, Kim KY, Terada M, Hatori M, Peltier ST, Panda S, Merkle A, Ellisman MH (2015) $\mathrm{X}$-ray microscopy as an approach to increasing accuracy and efficiency of serial block-face imaging for correlated light and electron microscopy of biological specimens. Microsc Microanal 21: 231-238.

Buytaert J, Goyens J, De Greef D, Aerts P, Dirckx J (2014) Volume shrinkage of bone, brain and muscle tissue in sample preparation for micro-CT and light sheet fluorescence microscopy (LSFM). Microsc Microanal 20: 1208-1217.

Buytaert JA, Johnson SB, Dierick M, Salih WH, Santi PA (2013) MicroCT versus sTSLIM 3D imaging of the mouse cochlea. J Histochem Cytochem 61: 382-395.

Calder JD, Buttery L, Revell PA, Pearse M, Polak JM (2004) Apoptosis - a significant cause of bone cell death in osteonecrosis of the femoral head. J Bone Joint Surg Br 86: $1209-1213$.

Cardoso L, Fritton SP, Gailani G, Benalla M, Cowin SC (2013) Advances in assessment of bone porosity, permeability and interstitial fluid flow. J Biomech 46: 253-265.

Cardoso L, Herman BC, Verborgt O, Laudier D, Majeska RJ, Schaffler MB (2009) Osteocyte apoptosis controls activation of intracortical resorption in response to bone fatigue. J Bone Miner Res 24: 597-605.

Carter Y, Suchorab JL, Thomas CD, Clement JG, Cooper DML (2014) Normal variation in cortical osteocyte lacunar parameters in healthy young males. J Anat 225: 328-336.

Carter Y, Thomas CDL, Clement JG, Cooper DML (2013a) Femoral osteocyte lacunar density, volume and morphology in women across the lifespan. J Struct Biol 183: 519-526.

Carter Y, Thomas CDL, Clement JG, Peele AG, Hannah K, Cooper DM (2013b) Variation in osteocyte lacunar morphology and density in the human femur - a synchrotron radiation micro-CT study. Bone 52: 126-132.

Carzaniga R, Domart MC, Collinson LM, Duke E (2014) Cryo-soft X-ray tomography: a journey into the world of the native-state cell. Protoplasma 251: 449-458.

Cheng BX, Zhao SJ, Luo J, Sprague E, Bonewald LF, Jiang JX (2001) Expression of functional gap junctions and regulation by fluid flow in osteocyte-like MLO-Y4 cells. J Bone Miner Res 16: 249-259.

Ciani C, Sharma D, Doty SB, Fritton SP (2014) Ovariectomy enhances mechanical load-induced solute transport around osteocytes in rat cancellous bone. Bone 59: $229-234$.

Cloetens P, Ludwig W, Baruchel J, Van Dyck D, Van Landuyt J, Guigay JP, Schlenker M (1999) Holotomography: Quantitative phase tomography with micrometer resolution using hard synchrotron radiation X-rays. Appl Phys Lett 75: 2912-2914.
Coughlin TR, Voisin M, Schaffler MB, Niebur GL, McNamara LM (2015) Primary cilia exist in a small fraction of cells in trabecular bone and marrow. Calcif Tissue Int 96: 65-72.

Cowin SC, Moss-Salentijn L, Moss ML (1991) Candidates for the mechanosensory system in bone. $\mathrm{J}$ Biomech Eng 113: 191-197.

Cowin SC, Weinbaum S (1998) Strain amplification in the bone mechanosensory system. Am J Med Sci 316: 184-188.

Cowin SC, Weinbaum S, Zeng Y (1995) A case for bone canaliculi as the anatomical site of strain generated potentials. J Biomech 28: 1281-1297.

Cummings SR, Melton LJ (2002) Epidemiology and outcomes of osteoporotic fractures. Lancet 359: 17611767.

Dallas SL, Bonewald LF (2010) Dynamics of the transition from osteoblast to osteocyte. Ann N Y Acad Sci 1192: 437-443.

Dallas SL, Veno PA, Rosser JL, Barragan-Adjemian C, Rowe DW, Kalajzic I, Bonewald LF (2009) Time lapse imaging techniques for comparison of mineralization dynamics in primary murine osteoblasts and the late osteoblast/early osteocyte-like cell line MLO-A5. Cells Tissues Organs 189: 6-11.

Deerinck TJ, Bushong E, Lev-Ram V, Shu X, Tsien RY, Ellisman MH (2010) Enhancing serial block-face scanning electron microscopy to enable high resolution 3-D nanohistology of cells and tissues. Microsc Microanal 16: $1138-1139$.

Deligianni DD, Apostolopoulos CA (2008) Multilevel finite element modeling for the prediction of local cellular deformation in bone. Biomech Model Mechanobiol 7: 151-159.

Denk W, Horstmann H (2004) Serial block-face scanning electron microscopy to reconstruct threedimensional tissue nanostructure. PLoS Biol 2: e329.

Dierolf M, Menzel A, Thibault P, Schneider P, Kewish CM, Wepf R, Bunk O, Pfeiffer F (2010) Ptychographic $\mathrm{X}$-ray computed tomography at the nanoscale. Nature 467 : 436-439.

Dong P, Haupert S, Hesse B, Langer M, Gouttenoire PJ, Bousson V, Peyrin F (2014) 3D osteocyte lacunar morphometric properties and distributions in human femoral cortical bone using synchrotron radiation microCT images. Bone 60: 172-185.

Doty SB (1981) Morphological evidence of gapjunctions between bone cells. Calcif Tissue Int 33: 509-512.

Draenert ME, Draenert AI, Forriol F, Erler M, Kunzelmann KH, Hickel R, Draenert K (2012) Value and limits of $\mu-\mathrm{CT}$ for nondemineralized bone tissue processing. Microsc Res Tech 75: 416-424.

Ellisman MH, Johnson JR, Deerinck TJ, Bushong EA, Bouwer J, Rumachandra R, Siegel JS (2015) Highly conductive nanocomposite, biological and small molecule materials for enhanced resin conductivilty. In: Patentscope (W.I. Organization, ed), USA.

Ercius P, Alaidi O, Rames MJ, Ren G (2015) Electron tomography: a three-dimensional analytic tool for hard and soft materials research. Adv Mater 27: 5638-5663. 
Farach-Carson MC, Carson DD (2007) Perlecan - a multifunctional extracellular proteoglycan scaffold. Glycobiology 17: 897-905.

Fazzalari NL, Forwood MR, Smith K, Manthey BA, Herreen P (1998) Assessment of cancellous bone quality in severe osteoarthrosis: bone mineral density, mechanics, and microdamage. Bone 22: 381-388.

Feng JQ, Ward LM, Liu S, Lu Y, Xie Y, Yuan B, Yu X, Rauch F, Davis SI, Zhang S, Rios H, Drezner MK, Quarles LD, Bonewald LF, White KE (2006) Loss of DMP1 causes rickets and osteomalacia and identifies a role for osteocytes in mineral metabolism. Nat Genet 38: 1310-1315.

Ferretti M, Muglia MA, Remaggi F, Cane V, Palumbo C (1999) Histomorphometric study on the osteocyte lacunocanalicular network in animals of different species. II. Parallel-fibered and lamellar bones. Ital J Anat Embryol 104: 121-131.

Friedl P, Hubbell J, Livingston D, Mihich E (2012) Twenty-third annual Pezcoller Symposium: Engineering influences in cancer research. Cancer Res 72: 841-844.

Fritton SP, Weinbaum S (2009) Fluid and solute transport in bone: flow-induced mechanotransduction. Annu Rev Fluid Mech 41: 347-374.

Frost HM, Jee WS (1992) On the rat model of human osteopenias and osteoporoses. Bone Miner 18: 227-236.

Gilbert SF (2000) Osteogenesis: The development of bones. In: Developmental biology, Sinauer Associates.

Goldsbury CS, Scheuring S, Kreplak L (2009) Introduction to atomic force microscopy (AFM) in biology. Curr Protoc Protein Sci, Chapter 58: 17.7:17.7.1-17.7.19.

Hafez A, Squires R, Pedracini A, Joshi A, Seegmiller RE, Oxford JT (2015) Col11a1 regulates bone microarchitecture during embryonic development. J Dev Biol 3: 158-176.

Han Y, Cowin SC, Schaffler MB, Weinbaum S (2004) Mechanotransduction and strain amplification in osteocyte cell processes. Proc Natl Acad Sci USA 101: 16689-16694.

Harris KM, Perry E, Bourne J, Feinberg M, Ostroff L, Hurlburt J (2006) Uniform serial sectioning for transmission electron microscopy. J Neurosci 26: 12101 12103.

Haugh MG, Vaughan TJ, McNamara LM (2015) The role of integrin alpha(V)beta(3) in osteocyte mechanotransduction. J Mech Behav Biomed Mater 42: 67-75.

Heino TJ, Kurata K, Higaki H, Vaananen HK (2009) Evidence for the role of osteocytes in the initiation of targeted remodeling. Technol Health Care 17: 49-56.

Henderson R, Sali A, Baker ML, Carragher B, Devkota B, Downing KH, Egelman EH, Feng Z, Frank J, Grigorieff N, Jiang W, Ludtke SJ, Medalia O, Penczek PA, Rosenthal PB, Rossmann MG, Schmid MF, Schroder GF, Steven AC, Stokes DL, Westbrook JD, Wriggers W, Yang H, Young J, Berman HM, Chiu W, Kleywegt GJ, Lawson CL (2012) Outcome of the first electron microscopy validation task force meeting. Structure 20: 205-214.

Hesse B, Langer M, Varga P, Pacureanu A, Dong P, Schrof S, Mannicke N, Suhonen H, Olivier C, Maurer P, Kazakia GJ, Raum K, Peyrin F (2014) Alterations of mass density and 3D osteocyte lacunar properties in bisphosphonate-related osteonecrotic human jaw bone, a synchrotron microCT study. PloS One 9: e88481.
Hesse B, Varga P, Langer M, Pacureanu A, Schrof S, Mannicke N, Suhonen H, Maurer P, Cloetens P, Peyrin F, Raum K (2015) Canalicular network morphology is the major determinant of the spatial distribution of mass density in human bone tissue: evidence by means of synchrotron radiation phase-contrast nano-CT. J Bone Miner Res 30: 346-356.

Hirose S, Li M, Kojima T, de Freitas PH, Ubaidus S, Oda K, Saito C, Amizuka N (2007) A histological assessment on the distribution of the osteocytic lacunar canalicular system using silver staining. J Bone Miner Metab 25: 374-382.

Holler M, Diaz A, Guizar-Sicairos M, Karvinen P, Färm E, Härkönen E, Ritala M, Menzel A, Raabe J, Bunk O (2014) X-ray ptychographic computed tomography at $16 \mathrm{~nm}$ isotropic 3D resolution. Sci Rep 4: 3857.

Holmbeck K, Bianco P, Pidoux I, Inoue S, Billinghurst RC, Wu W, Chrysovergis K, Yamada S, Birkedal-Hansen H, Poole AR (2005) The metalloproteinase MT1-MMP is required for normal development and maintenance of osteocyte processes in bone. J Cell Sci 118: 147-156.

Hoshaw SJ, Fyhrie DP, Takano Y, Burr DB, Milgrom C (1997) A method suitable for in vivo measurement of bone strain in humans. J Biomech 30: 521-524.

Jaiprakash A, Prasadam I, Feng JQ, Liu Y, Crawford R, Xiao Y (2012) Phenotypic characterization of osteoarthritic osteocytes from the sclerotic zones: a possible pathological role in subchondral bone sclerosis. Int J Biol Sci 8: 406417.

Jemielita M, Taormina MJ, DeLaurier A, Kimmel CB, Parthasarathy R (2013) Comparing phototoxicity during the development of a zebrafish craniofacial bone using confocal and light sheet fluorescence microscopy techniques. J Biophotonics 6: 920-928.

Jesse S, Kumar A, Arruda TM, Kim Y, Kalinin SV, Ciucci F (2012) Electrochemical strain microscopy: Probing ionic and electrochemical phenomena in solids at the nanometer level. MRS Bulletin 37: 651-658.

Jones CW, Smolinski D, Keogh A, Kirk TB, Zheng MH (2005) Confocal laser scanning microscopy in orthopaedic research. Prog Histochem Cytochem 40: 1-71.

Jordan GR, Loveridge N, Power J, Clarke MT, Parker M, Reeve J (2003) The ratio of osteocytic incorporation to bone matrix formation in femoral neck cancellous bone: an enhanced osteoblast work rate in the vicinity of hip osteoarthritis. Calcif Tissue Int 72: 190-196.

Kamel-ElSayed SA, Tiede-Lewis LM, Lu Y, Veno PA, Dallas SL (2015) Novel approaches for two and three dimensional multiplexed imaging of osteocytes. Bone 76: 129-140.

Kamioka H (2015) Osteocyte bioimaging. J Oral Biosci 57: 61-64.

Kamioka H, Honjo T, Takano-Yamamoto T (2001) A three-dimensional distribution of osteocyte processes revealed by the combination of confocal laser scanning microscopy and differential interference contrast microscopy. Bone 28: 145-149.

Kamioka H, Kameo Y, Imai Y, Bakker AD, Bacabac RG, Yamada N, Takaoka A, Yamashiro T, Adachi T, KleinNulend J (2012) Microscale fluid flow analysis in a human osteocyte canaliculus using a realistic high-resolution 
image-based three-dimensional model. Integr Biol (Camb) 4: 1198-1206.

Kamioka H, Murshid SA, Ishihara Y, Kajimura N, Hasegawa T, Ando R, Sugawara Y, Yamashiro T, Takaoka A, Takano-Yamamoto T (2009) A method for observing silver-stained osteocytes in situ in $3-\mu \mathrm{m}$ sections using ultra-high voltage electron microscopy tomography. Microsc Microanal 15: 377-383.

Katsamenis OL, Chong HMH, Andriotis OG, Thurner PJ (2013) Load-bearing in cortical bone microstructure: Selective stiffening and heterogeneous strain distribution at the lamellar level. J Mech Behav Biomed Mater 17: 152-165.

Kawakami T, Takise S, Fuchimoto T, Kawata H (2009) Effects of masticatory movement on cranial bone mass and micromorphology of osteocytes and osteoblasts in developing rats. Asia Pac J Clin Nutr 18: 96-104.

Klein-Nulend J, Bakker AD, Bacabac RG, Vatsa A, Weinbaum S (2013) Mechanosensation and transduction in osteocytes. Bone 54: 182-190.

Klein-Nulend J, van der Plas A, Semeins CM, Ajubi NE, Frangos JA, Nijweide PJ, Burger EH (1995) Sensitivity of osteocytes to biomechanical stress in vitro. FASEB J 9: 441-445.

Knothe Tate ML, Adamson JR, Tami AE, Bauer TW (2004) The osteocyte. Int J Biochem Cell Biol 36: 1-8.

Knothe Tate ML, Niederer P, Knothe U (1998) In vivo tracer transport through the lacunocanalicular system of rat bone in an environment devoid of mechanical loading. Bone 22: 107-117.

Knothe Tate ML, Tami AEG, Bauer TW, Knothe U (2002) Micropathoanatomy of osteoporosis: Indications for a cellular basis of bone disease. Adv Osteoporotic Fract Manag 2: 9-14.

Knott G, Genoud C (2013) Is EM dead? J Cell Sci 126: 4545-4552.

Kogawa M, Wijenayaka AR, Ormsby RT, Thomas GP, Anderson PH, Bonewald LF, Findlay DM, Atkins GJ (2013) Sclerostin regulates release of bone mineral by osteocytes by induction of carbonic anhydrase 2. J Bone Miner Res 28: 2436-2448.

Kubek DJ, Gattone VH, 2nd, Allen MR (2010) Methodological assessment of acid-etching for visualizing the osteocyte lacunar-canalicular networks using scanning electron microscopy. Microsc Res Tech 73: 182-186.

Kufahl RH, Saha S (1990) A theoretical model for stress-generated fluid flow in the canaliculi-lacunae network in bone tissue. J Biomech 23: 171-180.

Kwon KY, Wang E, Chung A, Chang N, Lee SW (2009) Effect of salinity on hydroxyapatite dissolution studied by atomic force microscopy. J Phys Chem 113: 3369-3372.

Lai X, Price C, Modla S, Thompson WR, Caplan J, Kirn-Safran CB, Wang L (2015) The dependences of osteocyte network on bone compartment, age, and disease. Bone Res 3: 15009.

Lam SS, Martell JD, Kamer KJ, Deerinck TJ, Ellisman MH, Mootha VK, Ting AY (2015) Directed evolution of APEX2 for electron microscopy and proximity labeling. Nat Methods 12: 51-54.

Langer M, Pacureanu A, Suhonen H, Grimal Q, Cloetens P, Peyrin F (2012) X-Ray phase nanotomography resolves the 3D human bone ultrastructure. PloS One, 7: e35691.

Lee KL, Guevarra MD, Nguyen AM, Chua MC, Wang Y, Jacobs CR (2015) The primary cilium functions as a mechanical and calcium signaling nexus. Cilia 4: 7.

Li XD, Ominsky MS, Warmington KS, Morony S, Gong JH, Cao J, Gao Y, Shalhoub V, Tipton B, Haldankar R, Chen Q, Winters A, Boone T, Geng ZP, Niu QT, Ke HZ, Kostenuik PJ, Simonet WS, Lacey DL, Paszty C (2009) Sclerostin antibody treatment increases bone formation, bone mass, and bone strength in a rat model of postmenopausal osteoporosis. J Bone Miner Res 24: 578-588.

Lin Y, Xu S (2011) AFM analysis of the lacunarcanalicular network in demineralized compact bone. $\mathrm{J}$ Microsc 241: 291-302.

Liu Y, Cai H-L, Zelisko M, Wang Y, Sun J, Yan F, Ma F, Wang P, Chen QN, Zheng H, Meng X, Sharma P, Zhang Y, Li J (2014) Ferroelectric switching of elastin. Proc Natl Acad Sci USA 111: E2780-E2786.

Lloyd SA, Loiselle AE, Zhang Y, Donahue HJ (2014) Shifting paradigms on the role of connexin43 in the skeletal response to mechanical load. J Bone Miner Res 29: 275286.

Lucas MS, Günthert M, Gasser P, Lucas F, Wepf R (2012) Bridging microscopes: 3D correlative light and scanning electron microscopy of complex biological structures. Methods Cell Biol 111: 325-356.

Malone AM, Anderson CT, Tummala P, Kwon RY, Johnston TR, Stearns T, Jacobs CR (2007) Primary cilia mediate mechanosensing in bone cells by a calciumindependent mechanism. Proc Natl Acad Sci USA 104: 13325-13330.

Marotti G, Ferretti M, Remaggi F, Palumbo C (1995) Quantitative evaluation on osteocyte canalicular density in human secondary osteons. Bone 16: 125-128.

Martin RB (2000) Toward a unifying theory of bone remodeling. Bone 26: 1-6.

Martin RB (2002) Is all cortical bone remodeling initiated by microdamage? Bone 30: 8-13.

Marx V (2013) Neurobiology: Brain mapping in high resolution. Nature 503: 147-152.

Masedunskas A, Milberg O, Porat-Shliom N, Sramkova M, Wigand T, Amornphimoltham P, Weigert R (2012) Intravital microscopy: a practical guide on imaging intracellular structures in live animals. Bioarchitecture 2: 143-157.

McCreadie BR, Hollister SJ, Schaffler MB, Goldstein SA (2004) Osteocyte lacuna size and shape in women with and without osteoporotic fracture. J Biomech 37: 563-572.

McIntosh R, Nicastro D, Mastronarde D (2005) New views of cells in 3D: an introduction to electron tomography. Trends Cell Biol 15: 43-51.

McNamara LM, Majeska RJ, Weinbaum S, Friedrich V, Schaffler MB (2009) Attachment of osteocyte cell processes to the bone matrix. Anat Rec (Hoboken) 292: 355-363.

McNamara LM, Prendergast PJ (2007) Bone remodelling algorithms incorporating both strain and microdamage stimuli. J Biomech 40: 1381-1391. 
McNulty MS, Bedell VM, Greenwood TM, Craig TA, Ekker SC, Kumar R (2012) Expression of sclerostin in the developing zebrafish (Danio rerio) brain and skeleton. Gene Expr Patterns 12: 228-235.

Mikuni-Takagaki Y, Kakai Y, Satoyoshi M, Kawano E, Suzuki Y, Kawase T, Saito S (1995) Matrix mineralization and the differentiation of osteocyte-like cells in culture. J Bone Miner Res 10: 231-242.

Milgrom C, Finestone A, Hamel A, Mandes V, Burr D, Sharkey N (2004) A comparison of bone strain measurements at anatomically relevant sites using surface gauges versus strain gauged bone staples. J Biomech 37: 947-952.

Milovanovic P, Zimmermann EA, Hahn M, Djonic D, Puschel K, Djuric M, Amling M, Busse B (2013) Osteocytic canalicular networks: morphological implications for altered mechanosensitivity. ACS Nano 7: 7542-7551.

Miszkiewicz JJ (2016) Investigating histomorphometric relationships at the human femoral midshaft in a biomechanical context. J Bone Miner Metab 34: 179-192.

Mohun TJ, Weninger WJ (2012) Generation of volume data by episcopic three-dimensional imaging of embryos. Cold Spring Harb Protoc 2012: 681-682.

Motyl KJ, McCauley LK, McCabe LR (2012) Amelioration of type I diabetes-induced osteoporosis by parathyroid hormone is associated with improved osteoblast survival. J Cell Physiol 227: 1326-1334.

Mullen CA, Vaughan TJ, Voisin MC, Brennan MA, Layrolle P, McNamara LM (2014) Cell morphology and focal adhesion location alters internal cell stress. J R Soc Interface 11: 20140885.

Mullender MG, Tan SD, Vico L, Alexandre C, KleinNulend J (2005) Differences in osteocyte density and bone histomorphometry between men and women and between healthy and osteoporotic subjects. Calcif Tissue Int 77: 291-296.

Mullender MG, van der Meer DD, Huiskes R, Lips P (1996) Osteocyte density changes in aging and osteoporosis. Bone 18: 109-113.

Müller R (2009) Hierarchical microimaging of bone structure and function. Nat Rev Rheumatol 5: 373-381.

Neve A, Corrado A, Cantatore FP (2012) Osteocytes: central conductors of bone biology in normal and pathological conditions. Acta Physiol 204: 317-330.

Nguyen VH, Lemaire T, Naili S (2011) Influence of interstitial bone microcracks on strain-induced fluid flow. Biomech Model Mechanobiol 10: 963-972.

Nicolella DP, Bonewald LF, Moravits DE, Lankford J (2005) Measurement of microstructural strain in cortical bone. Eur J Morphol 42: 23-29.

Nicolella DP, Moravits DE, Gale AM, Bonewald LF, Lankford J (2006) Osteocyte lacunae tissue strain in cortical bone. J Biomech 39: 1735-1743.

Niedzwiedzki T, Filipowska J (2015) Bone remodeling in the context of cellular and systemic regulation: the role of osteocytes and the nervous system. J Mol Endocrinol 55: R23-R36.

Noble BS, Peet N, Stevens HY, Brabbs A, Mosley JR, Reilly GC, Reeve J, Skerry TM, Lanyon LE (2003) Mechanical loading: biphasic osteocyte survival and targeting of osteoclasts for bone destruction in rat cortical bone. Am J Physiol Cell Physiol 284: C934-C943.

Okada S, Yoshida S, Ashrafi SH, Schraufnagel DE (2002) The canalicular structure of compact bone in the rat at different ages. Microsc Microanal 8: 104-115.

Ominsky MS, Niu QT, Li CY, Li XD, Ke HZ (2014) Tissue-level mechanisms responsible for the increase in bone formation and bone volume by sclerostin antibody. J Bone Miner Res 29: 1424-1430.

Ong ACM, Wheatley DN (2003) Polycystic kidney disease - the ciliary connection. Lancet 361: 774-776.

Palumbo C, Palazzini S, Zaffe D, Marotti G (1990) Osteocyte differentiation in the tibia of newborn rabbit: an ultrastructural study of the formation of cytoplasmic processes. Acta Anat (Basel) 137: 350-358.

Peddie CJ, Collinson LM (2014) Exploring the third dimension: volume electron microscopy comes of age. Micron 61: 9-19.

Pennycook TJ, Lupini AR, Yang H, Murfitt MF, Jones L, Nellist PD (2015) Efficient phase contrast imaging in STEM using a pixelated detector. Part 1: Experimental demonstration at atomic resolution. Ultramicroscopy 151: 160-167.

Peyrin F, Dong P, Pacureanu A, Langer M (2014) Micro- and nano-CT for the study of bone ultrastructure. Curr Osteoporos Rep 12: 465-474.

Peyrin F, Salome M, Cloetens P, Laval-Jeantet AM, Ritman E, Rüegsegger P (1998) Micro-CT examinations of trabecular bone samples at different resolutions: 14, 7 and 2 micron level. Technol Health Care 6: 391-401.

Piekarski K, Munro M (1977) Transport mechanism operating between blood supply and osteocytes in long bones. Nature 269: 80-82.

Plotkin LI, Speacht TL, Donahue HJ (2015) Cx43 and mechanotransduction in bone. Curr Osteoporos Rep 13: 67-72.

Power J, Loveridge N, Rushton N, Parker M, Reeve J (2002) Osteocyte density in aging subjects is enhanced in bone adjacent to remodeling haversian systems. Bone 30: 859-865.

Qiu N, Xiao ZS, Cao L, Buechel MM, David V, Roan E, Quarles LD (2012) Disruption of Kif3a in osteoblasts results in defective bone formation and osteopenia. J Cell Sci 125: 1945-1957.

Qiu S, Rao DS, Palnitkar S, Parfitt AM (2002) Age and distance from the surface but not menopause reduce osteocyte density in human cancellous bone. Bone 31: 313-318.

Qiu SJ, Rao DS, Palnitkar S, Parfitt AM (2003) Reduced iliac cancellous osteocyte density in patients with osteoporotic vertebral fracture. J Bone Miner Res 18: 1657-1663.

Reilly GC, Haut TR, Yellowley CE, Donahue HJ, Jacobs CR (2003) Fluid flow induced PGE(2) release by bone cells is reduced by glycocalyx degradation whereas calcium signals are not. Biorheology 40: 591-603.

Reilly GC, Knapp HF, Stemmer A, Niederer P, Knothe Tate ML (2001) Investigation of the morphology of the lacunocanalicular system of cortical bone using atomic force microscopy. Ann Biomed Eng 29: 1074-1081. 
Remaggi F, Cane V, Palumbo C, Ferretti M (1998) Histomorphometric study on the osteocyte lacunocanalicular network in animals of different species. I. Woven-fibered and parallel-fibered bones. Ital J Anat Embryol 103: 145-155.

Ridha H, Almitani KH, Chamekh A, Toumi H, Tavares JMRS (2015) A theory for bone resorption based on the local rupture of osteocytes cells connections: A finite element study. Math Biosci 262: 46-55.

Riquelme MA, Burra S, Kar R, Lampe PD, Jiang JX (2015) Mitogen-activated protein kinase (MAPK) activated by prostaglandin E2 phosphorylates connexin 43 and closes osteocytic hemichannels in response to continuous flow shear stress. J Biol Chem 290: 28321-28328.

Rubin MA, Jasiuk I (2005) The TEM characterization of the lamellar structure of osteoporotic human trabecular bone. Micron 36: 653-664.

Russo CJ, Passmore LA (2014) Robust evaluation of 3D electron cryomicroscopy data using tilt-pairs. J Struct Biol 187: 112-118.

Salome M, Peyrin F, Cloetens P, Odet C, Laval-Jeantet AM, Baruchel J, Spanne P (1999) A synchrotron radiation microtomography system for the analysis of trabecular bone samples. Med Phys 26: 2194-2204.

Salter DM, Robb JE, Wright MO (1997) Electrophysiological responses of human bone cells to mechanical stimulation: evidence for specific integrin function in mechanotransduction. J Bone Miner Res 12: 1133-1141.

Sansalone V, Kaiser J, Naili S, Lemaire T (2013) Interstitial fluid flow within bone canaliculi and electrochemo-mechanical features of the canalicular milieu: A multi-parametric sensitivity analysis. Biomech Model Mechanobiol 12: 533-553.

Schneider P, Meier M, Wepf R, Müller R (2010) Towards quantitative 3D imaging of the osteocyte lacunocanalicular network. Bone 47: 848-858.

Schneider P, Meier M, Wepf R, Müller R (2011) Serial FIB/SEM imaging for quantitative 3D assessment of the osteocyte lacuno-canalicular network. Bone 49: 304-311.

Schneider P, Ruffoni D, Larsson D, Chiapparini I, Müller R (2012) Image-based finite element models for the inestigation of osteocyte mechanotransduction. J Biomech 45 (S1): S436.

Schneider P, Stauber M, Voide R, Stampanoni M, Donahue LR, Müller R (2007) Ultrastructural properties in cortical bone vary greatly in two inbred strains of mice as assessed by synchrotron light based micro- and nano-CT. J Bone Miner Res 22: 1557-1570.

Shah AD, Shoback D, Lewiecki EM (2015a) Sclerostin inhibition: a novel therapeutic approach in the treatment of osteoporosis. Int J Womens Health 7: 565-580.

Shah FA, Johansson BR, Thomsen P, Palmquist A (2015b) Ultrastructural evaluation of shrinkage artefacts induced by fixatives and embedding resins on osteocyte processes and pericellular space dimensions. J Biomed Mater Res A 103: 1565-1576.

Sharma D, Ciani C, Marin PA, Levy JD, Doty SB, Fritton SP (2012) Alterations in the osteocyte lacunarcanalicular microenvironment due to estrogen deficiency. Bone 51: 488-497.
Shu X, Lev-Ram V, Deerinck TJ, Qi YC, Ramko EB, Davidson MW, Jin Y, Ellisman MH, Tsien RY (2011) A genetically encoded tag for correlated light and electron microscopy of intact cells, tissues, and organisms. PLoS Biol 9: e1001041.

Skinner RA (2003) Decalcification of bone tissue. In: Handbook of histology methods for bone and cartilage (An YH, Martin KL, eds) Springer Science and Business Media, pp 167-184.

Smith IO, Ren F, Baumann MJ, Case ED (2006) Confocal laser scanning microscopy as a tool for imaging cancellous bone. J Biomed Mater Res B Appl Biomater 79B: 185-192.

Spath A, Raabe J, Fink RH (2015) Confocal soft X-ray scanning transmission microscopy: setup, alignment procedure and limitations. J Synchrotron Radiat 22: 113118.

Staehling-Hampton K, Proll S, Paeper BW, Zhao L, Charmley P, Brown A, Gardner JC, Galas D, Schatzman RC, Beighton P, Papapoulos S, Hamersma H, Brunkow ME (2002) A 52-kb deletion in the SOST-MEOX1 intergenic region on 17q12-q21 is associated with van Buchem disease in the Dutch population. Am J Med Genet 110: 144-152.

Sterck JG, Klein-Nulend J, Lips P, Burger EH (1998) Response of normal and osteoporotic human bone cells to mechanical stress in vitro. Am J Physiol 274: E1113-E1120.

Sugawara Y, Ando R, Kamioka H, Ishihara Y, Honjo T, Kawanabe N, Kurosaka H, Takano-Yamamoto T, Yamashiro T (2011) The three-dimensional morphometry and cell-cell communication of the osteocyte network in chick and mouse embryonic calvaria. Calcif Tissue Int 88: 416-424.

Takaoka A, Hasegawa T, Yoshida K, Mori H (2008) Microscopic tomography with ultra-HVEM and applications. Ultramicroscopy 108: 230-238.

Tanaka-Kamioka K, Kamioka H, Ris H, Lim SS (1998) Osteocyte shape is dependent on actin filaments and osteocyte processes are unique actin-rich projections. J Bone Miner Res 13: 1555-1568.

Tapia JC, Kasthuri N, Hayworth KJ, Schalek R, Lichtman JW, Smith SJ, Buchanan J (2012) High-contrast en bloc staining of neuronal tissue for field emission scanning electron microscopy. Nat Protoc 7: 193-206.

Tatsumi S, Ishii K, Amizuka N, Li MQ, Kobayashi T, Kohno K, Ito M, Takeshita S, Ikeda K (2007) Targeted ablation of osteocytes induces osteoporosis with defective mechanotransduction. Cell Metab 5: 464-475.

Teti A, Zallone A (2009) Do osteocytes contribute to bone mineral homeostasis? Osteocytic osteolysis revisited. Bone 44: 11-6.

Thompson WR, Modla S, Grindel BJ, Czymmek KJ, Kirn-Safran CB, Wang LY, Duncan RL, Farach-Carson MC (2011) Perlecan/Hspg2 deficiency alters the pericellular space of the lacunocanalicular system surrounding osteocytic processes in cortical bone. J Bone Miner Res 26: 618-629.

Tomkinson A, Reeve J, Shaw RW, Noble BS (1997) The death of osteocytes via apoptosis accompanies estrogen withdrawal in human bone. J Clin Endocrinol Metab 82: 3128-3135. 
Tong W, Glimcher MJ, Katz JL, Kuhn L, Eppell SJ (2003) Size and shape of mineralites in young bovine bone measured by atomic force microscopy. Calcif Tissue Int 72: $592-598$.

Usukura J, Yoshimura A, Minakata S, Youn D, Ahn J, Cho SJ (2012) Use of the unroofing technique for atomic force microscopic imaging of the intra-cellular cytoskeleton under aqueous conditions. J Electron Microsc (Tokyo) 61: 321-326.

Uzbekov RE, Maurel DB, Aveline PC, Pallu S, Benhamou CL, Rochefort GY (2012) Centrosome fine ultrastructure of the osteocyte mechanosensitive primary cilium. Microsc Microanal 18: 1430-1441.

van Hove RP, Nolte PA, Vatsa A, Semeins CM, Salmon PL, Smit TH, Klein-Nulend J (2009) Osteocyte morphology in human tibiae of different bone pathologies with different bone mineral density - Is there a role for mechanosensing? Bone 45: 321-329.

Varga P, Hesse B, Langer M, Schrof S, Männicke N, Suhonen H, Pacureanu A, Pahr D, Peyrin F, Raum K (2015) Synchrotron X-ray phase nano-tomography-based analysis of the lacunar-canalicular network morphology and its relation to the strains experienced by osteocytes in situ as predicted by case-specific finite element analysis. Biomech Model Mechanobiol 14: 267-282.

Varga P, Pacureanu A, Langer M, Suhonen H, Hesse B, Grimal Q, Cloetens P, Raum K, Peyrin F (2013) Investigation of the three-dimensional orientation of mineralized collagen fibrils in human lamellar bone using synchrotron X-ray phase nano-tomography. Acta Biomater 9: $8118-8127$.

Vashishth D, Verborgt O, Divine G, Schaffler MB, Fyhrie DP (2000) Decline in osteocyte lacunar density in human cortical bone is associated with accumulation of microcracks with age. Bone 26: 375-380.

Vatsa A, Breuls RG, Semeins CM, Salmon PL, Smit TH, Klein-Nulend J (2008) Osteocyte morphology in fibula and calvaria - Is there a role for mechanosensing? Bone 43: 452-458.

Vaughan TJ, Mullen CA, Verbruggen SW, McNamara LM (2015) Bone cell mechanosensation of fluid flow stimulation: a fluid-structure interaction model characterising the role integrin attachments and primary cilia. Biomech Model Mechanobiol 14: 703-718.

Vavouraki AI, Putnis CV, Putnis A, Koutsoukos PG (2008) An Atomic Force Microscopy study of the growth of calcite in the presence of sodium sulfate. Chem Geol 253: 243-251.

Veno PA, Nicolella DP, Kalaizic I, Rowe DW, Bonewald LF, Dallas SL (2007) Dynamic imaging in living calvaria reveals the motile properties of osteoblasts and osteocytes and suggests heterogeneity of osteoblasts in bone. J Bone Miner Res 22(S1): S13.

Verbruggen SW, Vaughan TJ, McNamara LM (2012) Strain amplification in bone mechanobiology: a computational investigation of the in vivo mechanics of osteocytes. J R Soc Interface 9: 2735-2744.

Verbruggen SW, Vaughan TJ, McNamara LM (2014) Fluid flow in the osteocyte mechanical environment: a fluid-structure interaction approach. Biomech Model Mechanobiol 13: 85-97.
Wacker I, Schroeder RR (2013) Array tomography. J Microsc 252: 93-99.

Wacker I, Spomer W, Hofmann A, Gengenbach U, Thaler M, Ness L, Brey P, Schroder RR (2015) On the road to large volumes in LM and SEM: New tools for array tomography. Microsc Microanal 21: 539-540.

Wang K, Sun W, Richie CT, Harvey BK, Betzig E, Ji N (2015a) Direct wavefront sensing for high-resolution in vivo imaging in scattering tissue. Nat Commun 6: 7276.

Wang L, Ciani C, Doty SB, Fritton SP (2004) Delineating bone's interstitial fluid pathway in vivo. Bone 34: 499-509.

Wang L, Dong J, Xian CJ (2015b) Strain amplification analysis of an osteocyte under static and cyclic loading: a finite element study. Biomed Res Int 2015: 376474.

Wang Y, McNamara LM, Schaffler MB, Weinbaum S (2007) A model for the role of integrins in flow induced mechanotransduction in osteocytes. Proc Natl Acad Sci USA 104: 15941-15946.

Weber M, Mickoleit M, Huisken J (2014) Chapter 11 Light sheet microscopy. In: Methods in cell biology (C.W. Jennifer, W. Torsten, eds), Academic Press, pp 193-215.

Webster DJ, Schneider P, Dallas SL, Müller R (2013) Studying osteocytes within their environment. Bone 54: 285-295.

Weinbaum S, Cowin SC, Zeng Y (1994) A model for the excitation of osteocytes by mechanical loading-induced bone fluid shear stresses. J Biomech 27: 339-360.

Weston AE, Armer HE, Collinson LM (2010) Towards native-state imaging in biological context in the electron microscope. J Chem Biol 3: 101-112.

White JG, Southgate E, Thomson JN, Brenner S (1986) The structure of the nervous system of the nematode Caenorhabditis elegans. Philos Trans R Soc Lond B Biol Sci 314: 1-340.

Wong SYP, Evans RA, Needs C, Dunstan CR, Hills E, Garvan J (1987) The pathogenesis of osteoarthritis of the hip. Evidence for primary osteocyte death. Clin Orthop Relat Res 214: 305-312.

Wronski TJ, Lowry PL, Walsh CC, Ignaszewski LA (1985) Skeletal alterations in ovariectomized rats. Calcif Tissue Int 37: 324-328.

Wu D, Schaffler MB, Weinbaum S, Spray DC (2013) Matrix-dependent adhesion mediates network responses to physiological stimulation of the osteocyte cell process. Proc Natl Acad Sci USA 110: 12096-12101.

Xiao Z, Dallas M, Qiu N, Nicolella D, Cao L, Johnson M, Bonewald L, Quarles LD (2011) Conditional deletion of Pkd1 in osteocytes disrupts skeletal mechanosensing in mice. FASEB J 25: 2418-2432.

$\mathrm{Xu} \mathrm{HY}, \mathrm{Gu}$ SM, Riquelme MA, Burra S, Callaway D, Cheng HY, Guda T, Schmitz J, Fajardo RJ, Werner SL, Zhao H, Shang P, Johnson ML, Bonewald LF, Jiang JX (2015) Connexin 43 channels are essential for normal bone structure and osteocyte viability. J Bone Miner Res 30: 436-448.

You J, Yellowley CE, Donahue HJ, Zhang Y, Chen Q, Jacobs CR (2000) Substrate deformation levels associated with routine physical activity are less stimulatory to bone cells relative to loading-induced oscillatory fluid flow. $\mathrm{J}$ Biomech Eng 122: 387-393. 
You LD, Weinbaum S, Cowin SC, Schaffler MB (2004) Ultrastructure of the osteocyte process and its pericellular matrix. Anat Rec A Discov Mol Cell Evol Biol 278: 505513.

You L, Cowin SC, Schaffler MB, Weinbaum S (2001) A model for strain amplification in the actin cytoskeleton of osteocytes due to fluid drag on pericellular matrix. J Biomech 34: 1375-1386.

Zarrinkalam MR, Mulaibrahimovic A, Atkins GJ, Moore RJ (2012) Changes in osteocyte density correspond with changes in osteoblast and osteoclast activity in an osteoporotic sheep model. Osteoporos Int 23: 1329-1336.

Zeng Y, Cowin SC, Weinbaum S (1994) A fiber matrix model for fluid flow and streaming potentials in the canaliculi of an osteon. Ann Biomed Eng 22: 280-292.

Zhang K, Barragan-Adjemian C, Ye L, Kotha S, Dallas M, Lu Y, Zhao S, Harris M, Harris SE, Feng JQ, Bonewald LF (2006) E11/gp38 selective expression in osteocytes: regulation by mechanical strain and role in dendrite elongation. Mol Cell Biol 26: 4539-4552.

Zhang R, Gong H, Zhu D, Ma R, Fang J, Fan Y (2015) Multi-level femoral morphology and mechanical properties of rats of different ages. Bone 76: 76-87.

\section{Discussion with Reviewers}

Peter Varga: Authors mention the importance of validation. I completely agree that this is an important, but at the same time very challenging step. In your view, how could these models be validated? What would be a meaningful and doable approach?

Author: Validation of sample preparation and imaging techniques is important, to make sure that results accurately reflect the tissues and cells being examined. Comparing tissue prepared for 'standard' electron microscopy (EM) and cryo-EM (Dubochet et al., 1988) would provide insight into changes that occur during sample preparation. Conventional EM preparation involves aldehyde fixation, a combination of heavy metal stains, dehydration, and resin embedding. As water is the main constituent of biological material and dehydration leads to material aggregation and consequent loss of structural information (Dubochet and Sartori Blanc, 2001), maintaining cells in their hydrated state is an advantage. Cryofixation by plunge or slam freezing removes heat quickly, forming vitreous ice, which minimises the amount of crystal damage and immobilises water molecules within milliseconds. The limited depth of vitrification (several $\mu \mathrm{m}$ ) makes the technique eminently suitable for small particles and thin parts of cells, but less good for tissue samples. A better alternative for bone tissue would be cryo-electron microscopy of vitreous sections (CEMOVIS) (Al-Amoudi et al., 2004) which - when combined with high-pressure freezing - eliminates the effects of chemical fixation, staining, and dehydration. CEMOVIS gives close-to-native-state results at excellent spatial resolutions (Bouchet-Marquis and Fakan, 2009). It must be remembered however, that cryo-EM methods can introduce cutting artefacts such as compression, and that the act of imaging with an electron beam can also affect the samples by thinning or 'bubbling' reviewed in (Mielanczyk et al. (2014).

Peter Varga: The general problem with osteocyte imaging is still the lack of data. At the same time, we need proper statistical description on this cell network. You mention the need for comparing healthy and pathological conditions. Would you agree with the statement that we first would need to identify the normal variation of these features in healthy condition (sex, age, tissue age, anatomical site, loading condition, etc.)?

Author: We agree that it is important to understand bone anatomy on different hierarchical levels during development and ageing, as well as in health and disease. An initial approach, using novel three-dimensional (3D) highresolution imaging techniques for the assessment of the osteocyte network and the surrounding lacuno-canalicular network, should encompass comparisons between healthy and pathological conditions for different anatomical sites, thus elucidating inter-individual and intra-individual variability. A significant amount of work has already been published to identify and quantify normal variation of anatomical features of bone at different hierarchical levels, thus a meta-analysis of these investigations would help to consolidate the current knowledge and strengthen the evidence collected, specifically in terms of the osteocyte and the lacuno-canalicular network. Standardised measures would facilitate these comparisons, and the collection of data would feed into computational models in the context of mechanobiology for instance. To date, most studies have been based on small numbers of samples. We suggest that the pre-clinical and clinical studies on sclerostin (Clarke, 2014) are a potential source of significant amounts of data and tissue samples for validation studies. Large numbers of human and animal subjects have been treated with antisclerostin antibodies (romosozumab and blosozumab), which have been found to increase bone formation and bone mineral density. We believe that analysis of this cohort would provide much valuable data.

Pascal Buenzli: The authors emphasise the role of the osteocyte network anatomy for the network's mechanosensing and mechanotransducing capabilities. Comparative tables such as Table 1 are invaluable tools to help understand this role. However, being still in an exploratory phase, the field has no accepted standard for measuring ON\&LCN properties. Could the authors briefly discuss what network properties they believe future studies should aim to report in order to start building a standard and ease comparative studies?

Author: Studies of network characteristics in the brain have related modifications in Alzheimer's disease and schizophrenia (Bullmore and Sporns, 2009). As in principle, it may be possible to relate changes in the properties of the osteocyte and the lacuno-canalicular network (ON\&LCN) to different pathological bone conditions, it is crucial therefore how (3D) spatial characteristics are subsequently quantified. To create a standard for measuring ON\&LCN properties, which will facilitate comparative studies, a consistent and well-defined set of quantitative measures for 
describing the ON\&LCN is needed, which is independent of the employed imaging methods and the experimental conditions that are required. The quantification should ideally be user-independent and automated to ensure consistency and efficiency, although some human interpretation and intervention will presumably remain necessary. There will always be a compromise between field of view and spatial resolution as well as acquisition time, data volume, and related financial implications.

A framework has been proposed for the quantitative characterisation of osteocyte lacunae and their 3D spatial relationships (Mader et al., 2013), which included shape, orientation, number density, spatial distribution, and alignment. This framework is a valuable first step and should be expanded to encompass finer cellular detail such as cell processes and ultrastructure. In a study, where the osteocyte network has been related to the surrounding bone mineral quality, measures related to connectivity and spatial distribution were compared for the ON, including canalicular details (Kerschnitzki et al., 2013). Dong and colleagues used automated quantification to extract 3D shape descriptors from synchrotron radiation-based microcomputed tomography data (Dong et al., 2014). Combining the measures in these studies and including ultrastructural detail would lead to a comprehensive standard data set. In summary, we believe that a quantitative framework should include, although not be limited to, detail such as; osteocyte number density, spatial distribution and alignment, microstructural information including cell, peri-cellular space and lacunar shape and volume as well as ultrastructural detail such as process and canaliculus dimensions and tortuosity.

BoneJ, an ImageJ (Schneider et al., 2012) plug-in designed specifically to provide standard measures for bone image analysis (Doube et al., 2010) in an easily accessible and open-source approach, has provided a significant contribution to the field.

\section{Additional References}

Al-Amoudi A, Norlen LPO, Dubochet J (2004) Cryo-electron microscopy of vitreous sections of native biological cells and tissues. J Struct Biol 148: 131-135.
Bouchet-Marquis C, Fakan S (2009) Cryoelectron microscopy of vitreous sections: a step further towards the native state. Methods Mol Biol 464: 425-439.

Bullmore E, Sporns O (2009) Complex brain networks: graph theoretical analysis of structural and functional systems. Nat Rev Neurosci 10: 186-198.

Clarke BL (2014) Anti-sclerostin antibodies: Utility in treatment of osteoporosis. Maturitas 78: 199-204.

Dong P, Pacureanu A, Zuluaga MA, Olivier C, Grimal Q, Peyrin F (2014) Quantification of the 3D morphology of the bone cell network from synchrotron micro-CT Images. Image Anal Stereol 33: 157-166.

Doube M, Klosowski MM, Arganda-Carreras I, Cordelieres FP, Dougherty RP, Jackson JS, Schmid B, Hutchinson JR, Shefelbine SJ (2010) BoneJ: Free and extensible bone image analysis in ImageJ. Bone 47: 10761079.

Dubochet J, Adrian M, Chang JJ, Homo JC, Lepault J, McDowall AW, Schultz P (1988) Cryo-electron microscopy of vitrified specimens. Q Rev Biophys 21: 129-228.

Dubochet J, Sartori Blanc N (2001) The cell in absence of aggregation artifacts. Micron 32: 91-99.

Kerschnitzki M, Kollmannsberger P, Burghammer M, Duda GN, Weinkamer R, Wagermaier W, Fratzl P (2013) Architecture of the osteocyte network correlates with bone material quality. J Bone Miner Res 28: 1837-1845.

Mader KS, Schneider P, Müller R, Stampanoni M(2013) A quantitative framework for the 3D characterization of the osteocyte lacunar system. Bone 57: 142-154.

Mielanczyk L, Matysiak N, Michalski M, Buldak R, Wojnicz R (2014) Closer to the native state. Critical evaluation of cryo-techniques for transmission electron microscopy: preparation of biological samples. Folia Histochem Cytobiol 52: 1-17.

Schneider CA, Rasband WS, Eliceiri KW (2012) NIH Image to Image J: 25 years of image analysis. Nat Methods 9: 671-675.

Editor's Note: Scientific Editor in charge of the paper: Juerg Gasser. 